\title{
A case study about the mass exclusion limits for the BSM vector resonances with the direct couplings to the third quark generation
}

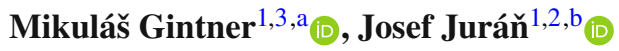 \\ ${ }^{1}$ Institute of Experimental and Applied Physics, Czech Technical University in Prague, Husova 240/5, 11000 Prague, Czech Republic \\ ${ }^{2}$ Institute of Physics, Silesian University in Opava, Bezručovo nám. 13, 74601 Opava, Czech Republic \\ ${ }^{3}$ Physics Department, University of Žilina, Univerzitná 1, 01026 Žilina, Slovakia
}

Received: 5 July 2019 / Accepted: 7 February 2020 / Published online: 21 February 2020

(C) The Author(s) 2020

\begin{abstract}
The upper bounds that the LHC measurements searching for heavy resonances beyond the Standard model set on the resonance production cross sections are not universal. They depend on various characteristics of the resonance under consideration, like its mass, spin, and its interaction pattern. Their validity is also limited by the assumptions and approximations applied to their calculations. The bounds are typically used to derive the mass exclusion limits for the new resonances. In our work, we address some of the issues that emerge when deriving the mass exclusion limits for the strongly coupled composite $S U(2)_{L+R}$ vector resonance triplet which would interact directly to the third quark generation only. We investigate the restrictions on the applicability of the generally used limit-obtaining procedure to this particular type of vector resonances. We demonstrate that, in this case, it is necessary to consider the bottom quark partonic contents of the proton. Eventually, we find the mass exclusion limits for this resonance triplet for some representative subsets of the parameter space.
\end{abstract}

\section{Introduction}

The existence of new particles, complementing the established Standard model (SM) spectrum, is predicted by all major scenarios of the SM extension. Should they be the supersymmetric partners to the SM fields or the composite resonances of new strong interactions, the discovery of the new particle(s) would provide undeniable evidence for beyond the SM physics. No wonder that the search for them has its rightful place in the ATLAS and CMS Collaboration's activities. Nevertheless, despite all their effort, no new parti-

\footnotetext{
a e-mail: gintner@fyzika.uniza.sk

b e-mail: josef.juran@utef.cvut.cz (corresponding author)
}

cle has been discovered so far. Actually, not even a significant disagreement with the SM predictions has been observed yet.

Facing the absence of the positive experimental input the available data can be used to set the exclusion limits on the parameters of the candidate BSM theories. First of all, the data can be translated into the excluded values of the masses of the sought-after new particles. Indeed, both Collaborations are making an effort to process their measurements into the form that can be used to establish the mass exclusion limits for the new particles of various kinds.

Obtaining the bound and, subsequently, the mass exclusion limit for the resonance of a particular BSM theory is a challenging task that requires the contributions of the experimental as well as theoretical communities. Since there is plenty of candidate theories with new particles of different properties, both communities seek to make the procedure as simple and general as possible. However, there are principal restrictions on how model-independent the analysis can become. They result from the specific properties of the sought-after resonance, like its spin or the absence of a certain decay channel. To calculate the mass exclusion limits correctly all significant model-imposed assumptions have to be identified and taken into account properly. Any simplifications, introduced in the calculations of the model cross sections, are welcomed. However, one has to remember that the simplifying assumptions can result in the reduction of the applicability of the obtained exclusion limits.

In their bounds producing analyses, the ATLAS and CMS Collaborations have focused on the on-shell direct production of the new particles in the LHC proton-proton collisions. There, the resonances are searched for in their various two-particle decay channels. The absence of the statistically significant deviation from the SM prediction in a given decay channel is then being translated into the upper bound on the production cross section of the BSM resonance multiplied by the relevant branching ratio. 
In order to work out the mass exclusion limit for a particular BSM model resonance theorists have to deliver the model's cross sections that are to be compared with the experimental upper bounds for the individual channels. If such a cross section exceeds the bound the resonance (and, thus, the model) is considered as being excluded by the experiment. When the resonance mass is a free parameter of the model, the regions of the excluded mass values can be established in this way.

In the strongly-interacting extensions of the SM, multiple new resonances emerge as the bound states of new stronglyinteracting fundamental fields. Since the forces responsible for the creation of the resonances are non-perturbative, a very limited information about the resonances can be obtained from the first principles. Thus, it is highly desirable to develop and use the effective description of the BSM bound state phenomenology; particularly, it would be the phenomenology of the bound states that might be observable at the LHC. Naturally, these usually include the lightest bound states of the given theory. While, in some scenarios, the spin-0 and spin-1 resonances are expected to be the lightest bound states [1-4], there are also light fermionic resonances predicted in others, see, e.g., [5-7].

Typical representatives of the strongly-interacting theories are the Technicolor model [8-10] and its extensions [1123]. More recent extra-dimensional theories [24-27] predict the Kaluza-Klein towers of new resonances of which the lowest lying ones might be observed at the LHC. The attractiveness of this development lies in the dual-description relation between the extra-dimensional weakly-interacting theories and the strongly-interacting models in four dimensions [28-32]. Closely related are the scenarios in which the Higgs appears as a composite pseudo-Goldstone boson of a strongly-coupled theory. Simple four-dimensional models, like the three-site [33], the four-site [34] and the effective composite Higgs model [35] can be used to characterize the main features of the emerging phenomenology.

In this paper, we focus on the strongly-interacting BSM vector resonances with a particular pattern of the interactions with the SM fermions: they couple directly to the top and bottom quarks only. Our ambition is to derive the exclusion limits for this kind of scenario and to understand the impact of its characteristic features on the applicability of the used simplifications. For this purpose, we use the effective Lagrangian that we developed and studied in [36-39] (the tBESS model).

Intuitively, it is natural to suspect that, due to its extraordinary mass, the top quark would play an outstanding role in the context of the new strong physics. The top mass being surprisingly close to the scale of the electroweak symmetry breaking (ESB) might be generated by the same mechanism as the masses of the electroweak gauge bosons [40,41]. This happens, for example, for the vector $\rho_{T}$ resonance of the
Extended Technicolor $[11,12]$. Yet, the mechanism behind the top mass could differ from that of ESB as it happens in the Topcolor Assisted Technicolor [21-23]. All these possibilities can result in the interactions of the vector resonances with the top quark that are different from the interactions with the light SM fermions. The neutral component of the new vector triplet can also mimic couplings of a $Z^{\prime}$ spin1 resonance [42,43] which has large couplings to $t$ and $b$, vanishing couplings to $W, Z$ and very small couplings to fermions of the first two generations. Regarding the enlarged couplings of the third generation quarks to the vector resonance triplet, similar features can be found in the Composite Higgs models [44-47] employing the idea of partial compositeness [48]. Since these models predict also new fermion resonances our effective description would apply to the cases when the effects of the fermions can be neglected. It is true that the Higgs boson in these models is a Goldstone boson rather than a generic singlet. Nevertheless, this does not have to change its low-energy dynamics dramatically.

Note that the effective description of the possible LHC phenomenology provided by the tBESS model is not necessarily in the rigorous one-to-one correspondence with any of the theories mentioned above. It can rather provide the effective description of the LHC phenomenology for proper subregions of the parameter space of various candidate theories. Thus, for example, while the richness of the resonance spectrum of many strongly-interacting theories far exceeds the particle contents of the tBESS model, this model might provide a satisfactory description of the LHC phenomenology for the parameter values when the majority of the resonances can be integrated out. In this way we can build a simplifying bridge for the application of the LHC experimental findings to the existing theories. The examples of this approach can be found in the literature, some of them even sharing certain features of the tBESS model. The effective description of the situation when the third quark generation couples extraordinarily to the new scalar and vector strong resonances was studied also in [42,49,50]. Another example of this approach when two, vector and axial-vector, $S U$ (2) triplets of the strong resonances were considered can be found in [51,52]. A "composite" scalar-vector system at the LHC was also studied in $[53,54]$. The phenomenology of a broad vector resonance in a more general formalism was studied in [55]. The broad vector resonance case decaying mainly to the third-generation quarks was also studied recently in [47].

In the tBESS effective model the Higgs sector is based on the non-linear sigma model with the $125-\mathrm{GeV} S U(2)_{L+R}$ scalar singlet complementing its non-linear triplet of the Nambu-Goldstone bosons. The vector resonances are present in the Lagrangian as an $S U(2)_{L+R}$ triplet. This setup fits the situation when the global $S U(2)_{L} \times S U(2)_{R}$ symmetry is broken down to $S U(2)_{L+R}$. 
The vector triplet is introduced as a gauge field via the hidden local symmetry approach [56]. Thus, the mass eigenstate representation of the vector resonance contains the admixture of EW gauge bosons. The gauge sector of this effective description is equivalent to the gauge sector of highlydeconstructed Higgsless model with three sites [33].

There are two specific features of the model that should be called attention to. First, the mass and decay widths of the vector resonances are entangled with the model's couplings. The vector resonance total width grows quite quickly with the resonance mass. The masses of the neutral and charged vector resonances are virtually degenerate when the resonance coupling $g^{\prime \prime}$ is much bigger than the $S U(2)_{L} \times U(1)_{Y}$ gauge couplings $\left(g, g^{\prime}\right)$.

Secondly, as advertised above, the direct interactions of the vector triplet with the third generation quarks only are admitted in the fermion sector. These interactions grow with $g^{\prime \prime}$ and can be introduced as flavor and chirality dependent, with no other interactions of the vector triplet with fermions in the flavor basis. ${ }^{1}$ However, the couplings of the vector resonance to the light SM fermions emerge in the mass eigenstate basis due to the mixing of the gauge bosons. These interactions (referred to as indirect couplings) are universal and suppressed by $1 / g^{\prime \prime}$. Thanks to the mixing-induced couplings, it is possible to produce the vector resonances also in the light-quark Drell-Yan processes at the LHC.

The experimental upper bounds, provided by the ATLAS and CMS Collaborations, are based on the narrow width resonance assumption. This assumption has its obvious benefits when deriving the experimental limits as well as for the calculation of the corresponding theoretical predictions. On the other hand, it restricts the scope of the method. It can be seen in our model. There are mass regions in the parametric space where the resonances are not so narrow and where the ratio exceeds the rule-of-thumb value of $10 \%$. We identify the regions of the parameter space where the method can be applied to the vector triplet under consideration.

In our paper [39], we did the analysis of the exclusion limits for our effective model with no direct couplings of the vector resonance triplet to the SM fermions. The universality of the indirect couplings justified the up-down quark only approximation of the proton partonic content under which the predictions of our model were calculated.

In the present work, the question about the role of the bquark partons in the vector boson production reappears due to the direct interactions between the vector triplet and the bottom quarks. Once more, one is tempted to ignore the tiny presence of the b-quarks in the proton, as we did in [39]. However, it will be shown that the direct couplings of the vector resonance with the bottom quark can even overwhelm

\footnotetext{
${ }^{1}$ Other sectors include self-interactions and the interactions with the SM EW gauge bosons and the Higgs boson.
}

the universal indirect interactions with fermions and, thus, compensate for the deficiency of the bottom quarks in the proton. In this paper, the first quark generation approximation used in [39] is upgraded to the all-sea-quark calculation.

In this paper, we establish the mass exclusion limits on the neutral and charged vector resonances of our model and observe their behavior. Because of the large dimensionality of the parameter space the full exclusion limit analysis would be a difficult task. Therefore, we determine the exclusion limits for selected subsets of the parameter space only. The limits are based on the most recent upper bounds on the LHC production cross sections times branching ratios published by the ATLAS and CMS Collaborations. To derive the exclusion limits we inspect and use all production mechanisms and decay channels relevant to our model for which the Collaborations published the upper bounds.

This paper is organized as follows. In Sect. 2, our model is briefly introduced and its phenomenology concerning its decay widths and branching ratios studied. Section 3 is concerned with the calculations of the model cross sections that are to be compared with the experimental upper bounds. Section 4 contains the analysis and calculations of the exclusion mass limits for both cases of the model: without and with the direct interactions to the third quark generation. The results of our work are summarized in the Conclusions section. Appendix A outlines the effective Lagrangian of our model.

\section{The effective Lagrangian and its phenomenology}

\subsection{The boson sectors}

The effective Lagrangian, we use in this paper, was studied in detail in our previous papers [36-38]. It can serve as an effective description of the LHC phenomenology of a hypothetical strongly interacting extension of the SM where the principal manifestation of this scenario would be the existence of a vector resonance triplet as a bound state of new strong interactions. The Lagrangian is built to respect the global $S U(2)_{L} \times S U(2)_{R} \times U(1)_{B-L} \times S U(2)_{H L S}$ symmetry of which the $S U(2)_{L} \times U(1)_{Y} \times S U(2)_{H L S}$ subgroup is also a local symmetry. The $S U(2)_{H L S}$ symmetry is an auxiliary gauge symmetry invoked to accommodate the $S U(2)_{L+R}$ triplet of new vector resonances. Each of the mentioned gauge groups is accompanied by its gauge coupling: $g$, $g^{\prime}$, and $g^{\prime \prime}$ stand for $S U(2)_{L}, U(1)_{Y}$ and $S U(2)_{L+R}$, respectively. Beside the scalar singlet representing the $125 \mathrm{GeV}$ Higgs boson and the hypothetical vector triplet, the effective Lagrangian is built out of the SM fields only. The effective Lagrangian itself can be found in [36-38] and its basic structure is summarized in Appendix A. 
The way the vector resonance triplet is introduced into the effective Lagrangian implies the mixing between the resonance and electroweak gauge boson fields. To decipher the physical content of the Lagrangian the gauge fields have to be transformed from the flavor to mass eigenstate basis. Note that the Greek letter $\rho$ will denote the vector boson resonance fields in the mass eigenstate basis. Consequently, $\rho^{ \pm}$ and $\rho^{0}$ stand for the charged and neutral members of the triplet, respectively.

When $g^{\prime \prime} \gg g, g^{\prime}$ the masses of the charged and neutral vector resonances are virtually degenerate. The leading order formula for the vector resonance mass reads

$M_{\rho}=\sqrt{\alpha} g^{\prime \prime} v / 2$,

where $\alpha$ is a dimensionless free parameter emerging in the effective Lagrangian and $v$ is the electroweak symmetry breaking scale. Usually, $\alpha$ is traded off for $M_{\rho}$ so that the latter can serve as one of the free parameters of the model. Our previous studies of the low-energy limits [36,37] as well as the Higgs-related limits and the unitarity limits [38] suggest that we should consider $M_{\rho} \geq 1 \mathrm{TeV}$ and $12 \leq g^{\prime \prime} \leq 25$. Following the conventions used in the formulation of our Lagrangian the naive perturbativity bound on $g^{\prime \prime}$ reads $g^{\prime \prime} / 2 \leq 4 \pi$.

In this paper, we calculate processes with the direct production of the vector resonances followed by their twoparticle decays. In the boson sector, the contributing triple couplings of the model include $\rho W W$ and $\rho W Z$ triple interactions. Their strengths are proportional to $1 / g^{\prime \prime}$. Note that the model contains neither of the $\rho Z Z, \rho Z \gamma, \rho \gamma \gamma$, and $\rho W \gamma$ vertices.

Additional bosonic triple vertices that could play a role in setting the mass exclusion limits include the $\rho W H$ and $\rho Z H$ vertices. Both couplings are proportional to the $a_{V}-a_{\rho}$ difference and they are also suppressed by the factor $1 / g^{\prime \prime}$. To a high precision, $a_{V}$ can be considered as a free pre-factor of the $H W W$ and $H Z Z$ vertices. The $H \rho^{0} \rho^{0}$ and $H \rho^{+} \rho^{-}$ couplings are virtually proportional to $a_{\rho}$. The corresponding interaction Lagrangian, along with the calculations of the LHC experimental limits for $a_{V}$ and $a_{\rho}$, can be found in [38].

Throughout this paper, we set $a_{V}=1$ (the SM case) and $a_{\rho}=0$ (no Higgs-to-vector resonance coupling). These values are consistent with the experimentally preferred points of the parameter space [38]. This choice that zeros the $H \rho^{0} \rho^{0}$ and $H \rho^{+} \rho^{-}$vertices and sets the $H W W$ and $H Z Z$ vertices to their SM form has no impact on our analysis. Neither it affects the results through the $\rho W H$ and $\rho Z H$ vertices.

To the leading order in $g^{\prime \prime}$ the vector resonance partial decay widths to $W^{+} W^{-}$and $W^{ \pm} Z$ read

$$
\begin{aligned}
\Gamma_{\rho \rightarrow V_{1} V_{2}=} & \Gamma_{\rho} \cdot\left[1+7\left(x_{1}^{2}+x_{2}^{2}\right)\right. \\
& \left.-26\left(x_{1}^{4}+x_{2}^{4}\right)-50 x_{1}^{2} x_{2}^{2}+\mathcal{O}\left(x^{6}\right)\right],
\end{aligned}
$$

where

$\Gamma_{\rho}=\frac{M_{\rho}}{48 \pi g^{\prime \prime 2}}\left(\frac{M_{\rho}}{v}\right)^{4}$,

$x_{1,2}=M_{1,2} / M_{\rho}$, and $V_{i}$ stands for either $W$ or $Z$.

The partial decay widths to the $W H$ and $W Z$ channels read

$\Gamma_{\rho^{ \pm} \rightarrow W^{ \pm} H}=4 \Gamma_{\rho} \cdot\left(a_{\rho}-a_{V}\right)^{2}\left[x_{W}^{4}+\mathcal{O}\left(x^{6}\right)\right]$,

and

$\Gamma_{\rho^{0} \rightarrow Z H}=4 \Gamma_{\rho} \cdot\left(a_{\rho}-a_{V}\right)^{2}\left[\left(2 x_{W}^{2}-x_{Z}^{2}\right)^{2}+\mathcal{O}\left(x^{6}\right)\right]$,

respectively. Within the $1-3 \mathrm{TeV}$ mass interval, the $W H$ and $W Z$ decay widths are four to six orders of magnitude smaller than the $W^{+} W^{-}$and $W^{ \pm} Z$ ones.

If there are no direct interactions of the vector resonance with fermions in the model, the widths of the fermion-related decay channels of the vector resonance are also quite negligible when compared to the $W^{+} W^{-}$and $W^{ \pm} Z$ decay channels. Thus, the total decay widths of the vector resonances, both neutral and charged, can be well approximated by the expression (3).

In Fig. 1, we depict how the vector resonance total width depends on the resonance mass and $g^{\prime \prime}$ using the full tree-level formulas for the calculations. The dashed lines correspond to

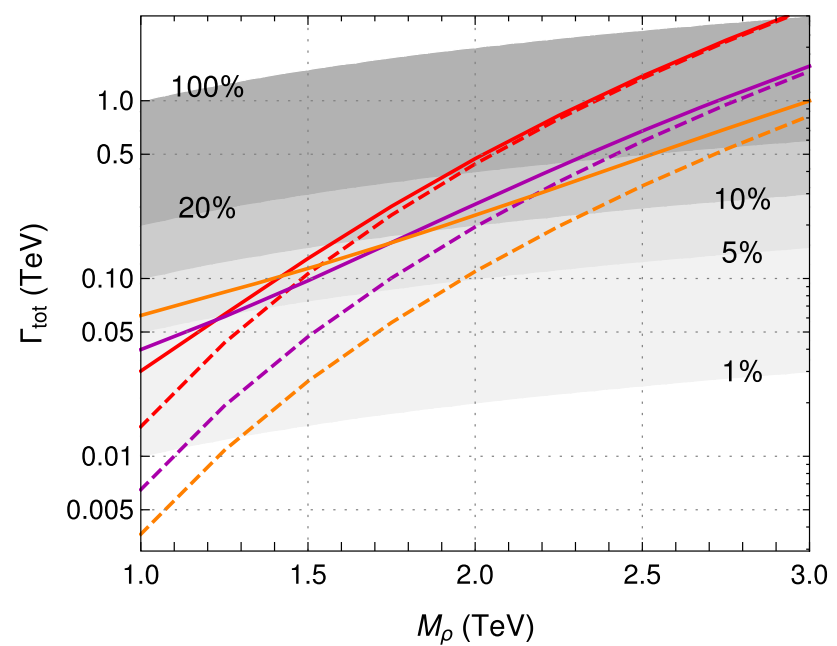

Fig. 1 The total decay width of the vector resonance as a function of the resonance mass. Top to bottom, the dashed lines correspond to $g^{\prime \prime}=12,18$, and 24 , respectively, when there is no direct interaction of the vector resonance to fermions. The solid lines representing the case of the direct interactions with $b_{L, R}=-0.1$, and $p=1$ unite with their dashed $g^{\prime \prime}$-counterparts at the upper right corner of the graph. Different shadings of the background indicate areas with different value ranges of $\Gamma_{\text {tot }} / M_{\rho}$. The curves do not distinguish between neutral and charged resonances 
the no-direct-interaction total widths when $g^{\prime \prime}=12,18$, and 24 , respectively. There is no visible distinction between the neutral and charged resonance curves in this graph. Different shadings of the background indicate regions with different values of the fatness of the resonance, $\Gamma_{\text {tot }} / M_{\rho}$. The graph also shows how the total width responds to the direct interactions. However, this part will be discussed in the following subsection.

\subsection{The fermion sector}

The only fermions considered in our effective model are those of the SM. Thus, the model describes the BSM situation when either non-SM fermionic fields are much heavier than $\mathcal{O}(1) \mathrm{TeV}$ vector resonances or their interactions make their existence irrelevant to our analysis.

Even if no direct couplings of the vector resonance fields to fermions are introduced in the flavor eigenstate basis the mixing between the vector resonance triplet and the electroweak gauge bosons induces the couplings between the vector resonance and fermions. These "indirect" couplings are proportional to $1 / g^{\prime \prime}$.

Nevertheless, the considered symmetry also admits the introduction of the direct interactions of the vector resonance with fermions. In fact, having grouped the right fermion fields into $S U(2)_{R}$ doublets the considered global symmetry of the model also allows for assigning different direct couplings to each of the $S U(2)_{L}$ and $S U(2)_{R}$ fermion doublets.

We assume in our model that the flavor basis vector triplet couples directly to the quarks of the third generation only and to none of the other SM fermions. This assumption is motivated by the anticipated extraordinary role of the top quark (and, perhaps, bottom quark as well) in new physics related to strong electroweak symmetry breaking. Similar interaction patterns can be found in various strong extensions of the SM including the partial compositeness and extradimensional scenarios. In our model, the free pre-factors for the couplings to the left and right top-bottom quark doublets are referred to as $b_{L}$ and $b_{R}$, respectively [36].

In the mass basis, the direct interactions also contaminate the couplings of $W$ and $Z$ to the top and bottom quarks. The contamination is proportional to $b_{L, R}$. It results in the experimental restrictions on $b_{L, R}$ from the EW precision measurements [37].

In the most general case of our model, an additional free pre-factor $p$ is introduced which serves to further modify the direct coupling to the right bottom quark. Assuming $0 \leq p \leq 1$, the $p$ parameter can be used to suppress the direct coupling to the right bottom quark relative to the direct coupling to the right top quark; $p=1$ leaves both interactions equal, $p=0$ turns off the right bottom quark interaction completely and maximally breaks the $S U(2)_{R}$ part of the Lagrangian symmetry down to $U(1)_{R 3}$. Recall that the
$S U(2)_{R}$ symmetry is broken by the weak hypercharge interactions anyway and, thus, the $S U(2)_{R}$ fermion doublets are not well justified once the global symmetry gets gauged.

This effective model was introduced and investigated in [36-38] as a modification of the so-called BESS model [57-59]. We refer to our model as the "top-BESS model" (tBESS) in order to recall its relation to its predecessor as well as to stress the special role of the top quark (or the third quark generation) in it. As was shown in $[36,37]$, these modifications also help relax some experimental restrictions burdening the original BESS model. Should we briefly summarize the conclusions reached in these studies, the absolute values of $b_{L, R}$ should not exceed 0.1 , roughly speaking. As far as $p$ is concerned, its most preferred value lies in the $0.2-0.3$ interval. However, the statistical preference of the interval with respect to any other value of $p$ between zero and one is weak.

Assuming massless fermions in the final state, the partial decay widths of the neutral vector resonance to the leptonic $\nu v$ and $\ell \ell$ channels read

$\Gamma_{\rho^{0} \rightarrow v v}=8 \Gamma_{\rho} \cdot\left(2 x_{W}^{2}-x_{Z}^{2}\right)^{2}$,

and

$\Gamma_{\rho^{0} \rightarrow \ell \ell}=8 \Gamma_{\rho} \cdot\left[x_{Z}^{4}+4\left(x_{Z}^{2}-x_{W}^{2}\right)^{2}\right]$,

respectively. Its partial decay widths to the light quark channels are

$\Gamma_{\rho^{0} \rightarrow u u}=24 \Gamma_{\rho} \cdot \frac{17 x_{Z}^{4}+20 x_{W}^{4}-28 x_{Z}^{2} x_{W}^{2}}{9}$,

and

$\Gamma_{\rho^{0} \rightarrow d d}=24 \Gamma_{\rho} \cdot \frac{5 x_{Z}^{4}+20 x_{W}^{4}-16 x_{Z}^{2} x_{W}^{2}}{9}$,

respectively. The corresponding partial decay widths of the charged vector resonance read

$3 \Gamma_{\rho^{ \pm} \rightarrow \nu \ell}=\Gamma_{\rho^{ \pm} \rightarrow u d}=48 \Gamma_{\rho} \cdot x_{W}^{4}$.

The partial widths of the decay channels with the top and bottom quarks in the final state are sensitive to the free parameters $b_{L, R}$ and $p$. They originate from the intertwining of the direct and indirect interactions. The $t t$ partial width reads

$$
\begin{aligned}
\Gamma_{\rho^{0} \rightarrow t t}= & \frac{M_{\rho}}{8 \pi} \beta_{t}\left\{\left[\left(g_{\rho t t}^{L}\right)^{2}+\left(g_{\rho t t}^{R}\right)^{2}\right]\left(1-x_{t}^{2}\right)\right. \\
& \left.+6 g_{\rho t t}^{L} g_{\rho t t}^{R} x_{t}^{2}\right\}
\end{aligned}
$$


where $\beta_{t}=\sqrt{1-4 x_{t}^{2}}, x_{t}=M_{t} / M_{\rho}$, and

$g_{\rho t t}^{L}=-\frac{b_{L}}{4} g^{\prime \prime}+\frac{2}{g^{\prime \prime}}\left[\left(\frac{2}{3}-b_{L}\right) \frac{M_{W}^{2}}{v^{2}}+\frac{1}{3} \frac{M_{Z}^{2}}{v^{2}}\right]$,

$g_{\rho t t}^{R}=-\frac{b_{R}}{4} g^{\prime \prime}+\frac{2}{g^{\prime \prime}}\left(\frac{4}{3}-b_{R}\right)\left(\frac{M_{Z}^{2}}{v^{2}}-\frac{M_{W}^{2}}{v^{2}}\right)$.

To the leading order in $g^{\prime \prime}$, the partial width (11) reads

$\Gamma_{\rho^{0} \rightarrow t t}=\frac{3}{8} \Gamma_{\rho} \cdot g^{\prime \prime 4}\left[\left(b_{L}^{2}+b_{R}^{2}\right) x_{v}^{4}+\mathcal{O}\left(x^{6}\right)\right]$,

where $x_{v}=v / M_{\rho}$ and $\mathcal{O}\left(x^{6}\right)$ represents any terms proportional to $x_{v}^{m} x_{t}^{n} x_{W}^{p} x_{Z}^{q}$ such that $m+n+p+q \geq 6$.

When the bottom mass is neglected the $b b$ partial width reads

$\Gamma_{\rho^{0} \rightarrow b b}=\frac{M_{\rho}}{8 \pi}\left[\left(g_{\rho b b}^{L}\right)^{2}+\left(g_{\rho b b}^{R}\right)^{2}\right]$

where

$g_{\rho b b}^{L}=\frac{b_{L}}{4} g^{\prime \prime}+\frac{2}{g^{\prime \prime}}\left[\left(b_{L}-\frac{4}{3}\right) \frac{M_{W}^{2}}{v^{2}}+\frac{1}{3} \frac{M_{Z}^{2}}{v^{2}}\right]$,

$g_{\rho b b}^{R}=p^{2} \frac{b_{R}}{4} g^{\prime \prime}+\frac{2}{g^{\prime \prime}}\left(p^{2} b_{R}-\frac{2}{3}\right)\left(\frac{M_{Z}^{2}}{v^{2}}-\frac{M_{W}^{2}}{v^{2}}\right)$.

To the leading order in $g^{\prime \prime}$, the partial width (15) reads

$\Gamma_{\rho^{0} \rightarrow b b}=\frac{3}{8} \Gamma_{\rho} \cdot g^{\prime \prime 4}\left(b_{L}^{2}+p^{4} b_{R}^{2}\right) x_{v}^{4}$.

Since we ignore the bottom quark mass there are no higher order corrections above $\mathcal{O}\left(x^{4}\right)$ to this expression.

Finally, the $t b$ partial width is

$\Gamma_{\rho^{ \pm} \rightarrow t b}=\frac{M_{\rho}}{8 \pi}\left[\left(g_{\rho t b}^{L}\right)^{2}+\left(g_{\rho t b}^{R}\right)^{2}\right]\left(1-3 x_{t}^{2} / 2+x_{t}^{6} / 2\right)$,

where

$g_{\rho t b}^{L}=-\frac{b_{L}}{2 \sqrt{2}} g^{\prime \prime}+\frac{2 \sqrt{2}}{g^{\prime \prime}}\left(1-b_{L}\right) \frac{M_{W}^{2}}{v^{2}}$,

$g_{\rho t b}^{R}=-p \frac{b_{R}}{2 \sqrt{2}} g^{\prime \prime}$.

To the leading order in $g^{\prime \prime}$, the partial width (19) reads

$\Gamma_{\rho^{ \pm} \rightarrow t b}=\frac{3}{4} \Gamma_{\rho} \cdot g^{\prime \prime 4}\left[\left(b_{L}^{2}+p^{2} b_{R}^{2}\right) x_{v}^{4}+\mathcal{O}\left(x^{6}\right)\right]$.

\subsection{The branching ratios}

If the vector resonance triplet does not interact with the fermions directly $\left(b_{L, R}=0\right)$, the branching ratios of all decay channels under consideration do not depend on $g^{\prime \prime}$. This is because all relevant decay widths are proportional to $\left(1 / g^{\prime \prime}\right)^{2}$. The no direct interaction branching ratios of the neutral as well as charged vector resonances are plotted in Fig. 2. The upper graph depicts curves for $\rho^{0} \rightarrow W^{+} W^{-}, t \bar{t}$, and $b \bar{b}$. The lower graph shows the curves for the $\rho^{+} \rightarrow W^{+} Z$ and $t \bar{b}$ channels. In addition, the upper and lower graphs contain plots of $\delta \Gamma_{0} / \Gamma_{\text {tot }}^{(0)}$ and $\delta \Gamma_{+} / \Gamma_{\text {tot }}^{(+)}$, respectively, where

$\delta \Gamma_{0}=\Gamma_{Z H}+3 \Gamma_{\nu v}+3 \Gamma_{\ell \ell}+2 \Gamma_{u u}+2 \Gamma_{d d}$,

$\delta \Gamma_{+}=\Gamma_{W H}+3 \Gamma_{v \ell}+2 \Gamma_{u d}$,

and

$\Gamma_{\text {tot }}^{(0)}=\Gamma_{W W}+\Gamma_{t t}\left(b_{L}, b_{R}\right)+\Gamma_{b b}\left(b_{L}, p^{2} b_{R}\right)+\delta \Gamma_{0}$,
$\Gamma_{\text {tot }}^{(+)}=\Gamma_{W Z}+\Gamma_{t b}\left(b_{L}, p b_{R}\right)+\delta \Gamma_{+}$,
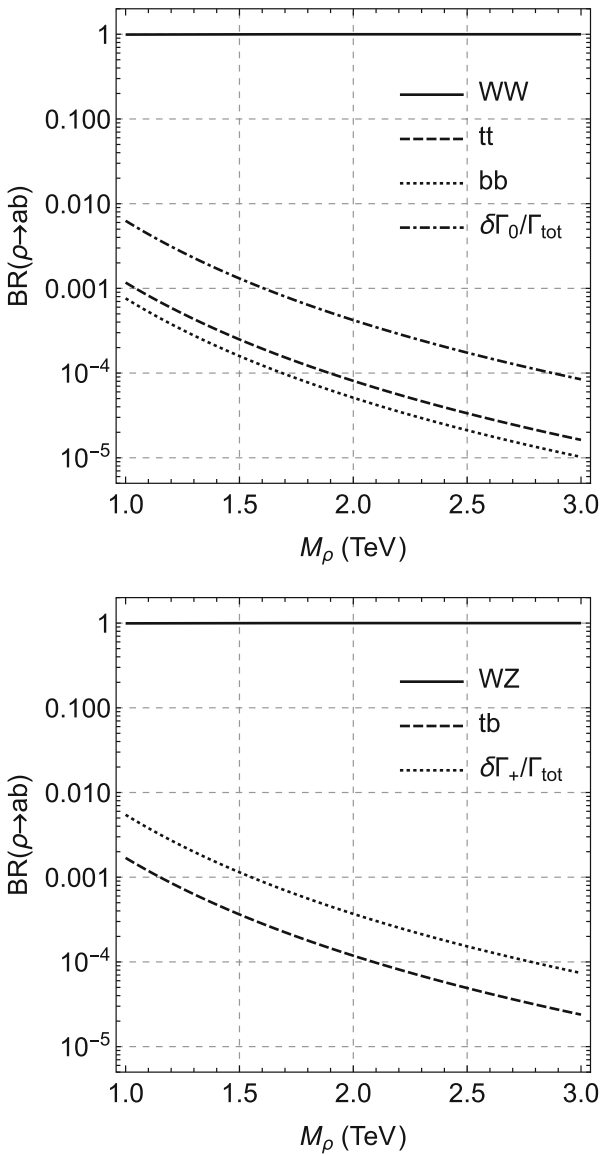

Fig. 2 The branching ratios of the neutral (top) and charged (bottom) vector resonances as functions of the resonance mass when there are no direct interactions of the resonance triplet with the top and bottom quarks 


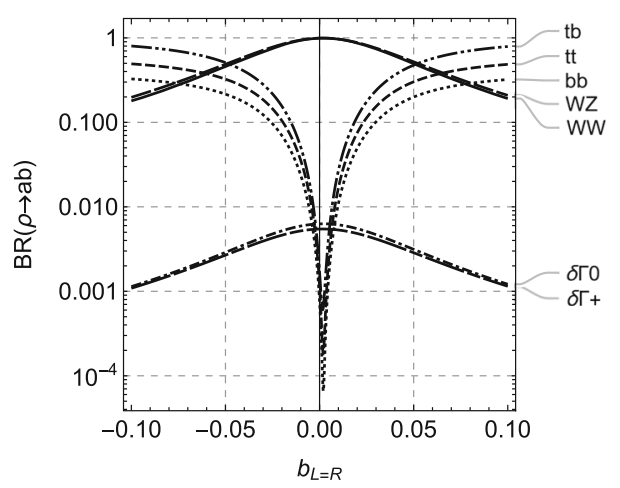

Fig. 3 The branching ratios of the neutral and charged vector resonances as functions of $b_{L=R} \equiv b_{L}=b_{R}$ when $M_{\rho}=1 \mathrm{TeV}, g^{\prime \prime}=18$, and $p=0.75$. The labels $\delta \Gamma 0$ and $\delta \Gamma+$ indicate the branching ratios corresponding to the decay widths given in the Eqs. (23) and (24), respectively

where we have assumed the same decay widths for the corresponding decay channels across all generations of leptons as well as across the first two generations of quarks. The CKM matrix is set to unity throughout the paper.

Now, let us consider the situation when the vector resonance triplet interacts directly with the third quark generation. It means that some or all of the $b_{L}, b_{R}, p$ parameters assume non-zero values. The five parameters of interest that can be varied independently, $M_{\rho}, g^{\prime \prime}$, and $b_{L}, b_{R}, p$, are too many for displaying the BR's behavior in a single plot. However, it is instructive to show BR's for the neutral and charged resonances as functions of their direct interactions to fermions when $b_{L}=b_{R} \equiv b_{L=R}$ and for a specific choice of the values of other parameters. Namely, we choose $M_{\rho}=1 \mathrm{TeV}, g^{\prime \prime}=18$, and $p=0.75$. The corresponding graph can be found in Fig. 3.

As expected, the Fig. 3 behavior of the branching ratios in the vicinity of $b_{L}=b_{R}=0$ corresponds well to that observed in Fig. 2. When $\left|b_{L=R}\right| \lesssim 10^{-2}$ the vector resonance decays are strongly dominated by the vector boson channels. The remaining channels contribute less than $1 \%$ to the total decay widths. In addition, in this region, the combined effect of the direct and indirect interactions of the vector resonance with the top and bottom quarks pulls the branching ratios of $\rho \rightarrow t t, b b, t b$ even below those of the light fermions. Actually, there are non-zero values of $b_{L, R}$ for which the direct and indirect interactions cancel each other out and the vector resonances cease to decay to ${ }^{2} t t, b b$, and $t b$. The glimpse of this effect can be seen in Fig. 3 where the minimum of the $t t, b b, t b$ curves is shifted slightly to the right of $b_{L=R}=0$, namely at about $b_{L=R}=0.002$.

\footnotetext{
2 The effect of the cancellation was studied in detail in our paper [36]. We nicknamed the parameter space region where the negative interference of the direct and indirect interactions suppressed the productions of $t \bar{t}, b \bar{b}$, and $t \bar{b} / b \bar{t}$ as "the Death Valley".
}
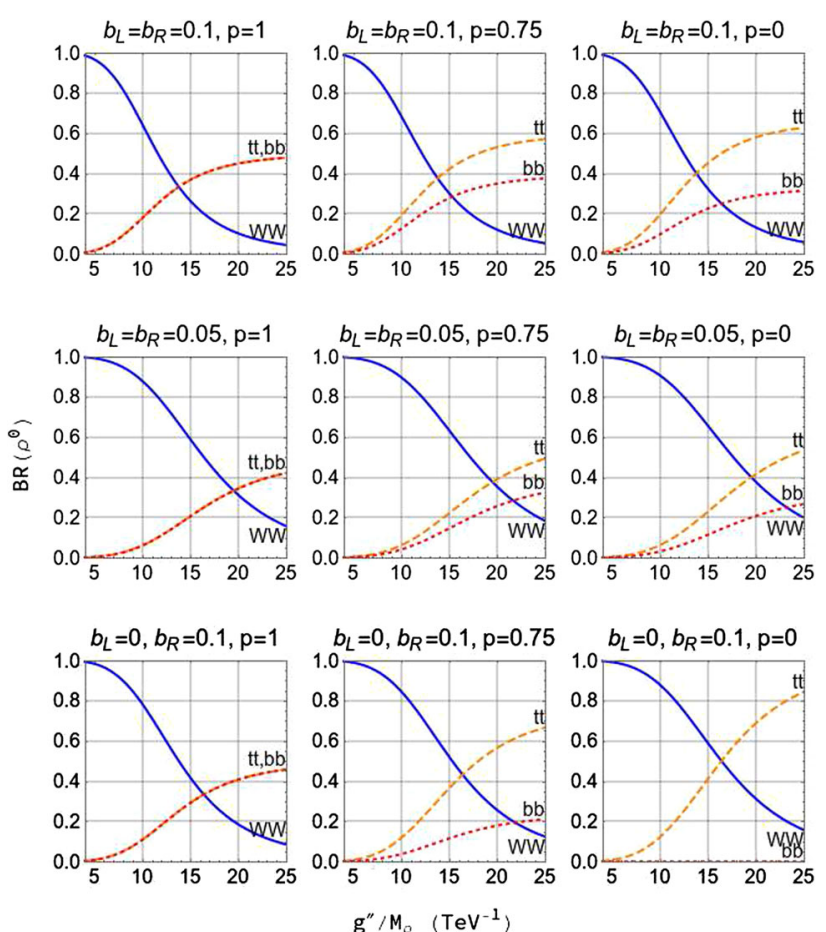

Fig. 4 The principal branching ratios of the neutral vector resonance as functions of $g^{\prime \prime} / M_{\rho}$ in the approximation of the leading order in $g^{\prime \prime}$ and $x_{a}=M_{a} / M_{\rho}=0$, where $a=W, Z, t$. The blue solid line stands for the $W W$ channel, the orange dashed line for the $t t$ channel, and the red dotted line represents the $b b$ channel

The branching ratios of the $t t, b b$, and $t b$ channels grow fast with the increasing $\left|b_{L=R}\right|$. It results from the unleashing the large contributions of the first terms of the couplings (12), (13), (16), (17), (20), and (21). These branching ratios reach the branching ratios of the light fermion channels at about $\left|b_{L, R}\right| \approx 0.01$ and they become comparable with the gauge boson ratios at about $\left|b_{L, R}\right| \approx 0.05$. Of course, the exact numbers depend on the values of the model parameters. Figure 3 just illustrates this model's behavior.

As the direct interactions grow stronger, the branching ratios of the $t t, b b$, and $t b$ channels become dependent on the $g^{\prime \prime} / M_{\rho}$ ratio only. This can be seen in the leading-order-in- $g^{\prime \prime}$ formulas for $t t, b b$, and $t b$ channels when $x_{a}=M_{a} / M_{\rho}=0$ for all relevant final state particles. These approximations introduce deviations from the exact branching ratios at the level of $x_{W, Z, t}^{2}$ and higher in the leading-order-in - $g^{\prime \prime}$ terms. The branching ratios of the principal decay channels in this approximation are displayed in Fig. 4 (the neutral resonance) and Fig. 5 (the charged resonance).

Nevertheless, the calculations of the mass exclusion limits in this paper are performed using the exact expressions for the decay widths and branching ratios. The approximations discussed above are meant to provide a better insight into the analysis and a better understanding of the results. 

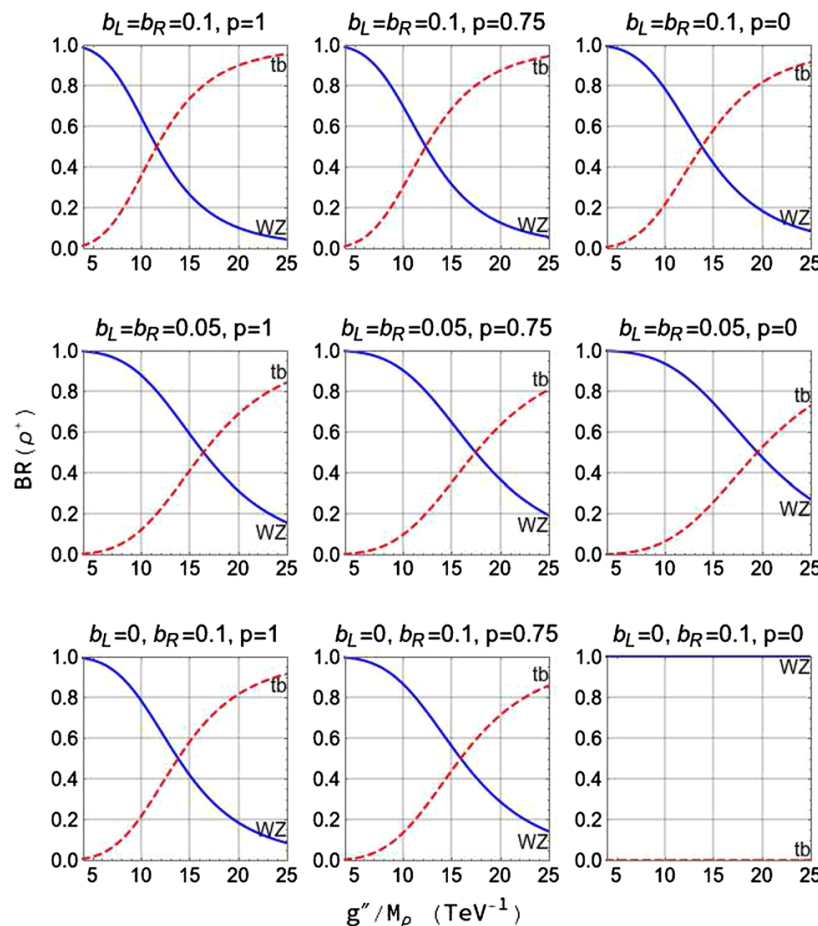

Fig. 5 The principal branching ratios of the charged vector resonance as functions of $g^{\prime \prime} / M_{\rho}$ in the approximation of the leading order in $g^{\prime \prime}$ and $x_{a}=M_{a} / M_{\rho}=0$, where $a=W, Z, t$. The blue solid line stands for the $W Z$ channel and the red dashed line represents the $t b$ channel

\section{Cross section calculations}

In principle, the current exclusion limits on the masses of new resonances result from the comparison of the upper experimental bounds for the resonance $s$-channel production cross sections to the model's predictions for this observable. The upper bounds are calculated by the ATLAS and CMS Collaborations using their data for various final states of various decay channels of the vector resonances. Consequently, the provided upper bounds restrict the on-shell cross sections $\sigma(p p \rightarrow \rho X \rightarrow a b X)$. Of course, the assumptions and procedures used in the model's prediction calculations have to comply with those used by the Collaborations in obtaining the upper bounds.

As long as the decay width of the produced resonance is not too wide for its mass, the cross section $\sigma(p p \rightarrow$ $\rho X \rightarrow a b X)$ can be conveniently approximated by the Narrow Width Approximation (NWA) formula

$\sigma(p p \rightarrow a b X)=\sigma_{\text {prod }}(p p \rightarrow \rho X) \times \mathrm{BR}(\rho \rightarrow a b)$,

where $\sigma_{\text {prod }}$ is the on-shell cross section for the vector resonance production, and $\operatorname{BR}(\rho \rightarrow a b)$ is the branching ratio for the vector resonance decay channel $\rho \rightarrow a b$. It is generally expected that the approximation (27) works well when $\Gamma_{\text {tot }} / M_{\rho} \lesssim 10 \%$. One should also remember that the NWA

ignores the signal-background interference effects. The influence of these effects on the precision of the approximation have been inspected in [60].

The experimental upper bounds have been delivered and updated continually by both Collaborations as, over the recent years, the amount of the data collected grew and no signs of new particles emerged. The collaborations worked out and published the upper bounds not only for the individual decay channels but also for their combinations. In addition, for the $W W$ and $W Z$ channels, they were able to distinguish between the Drell-Yan (DY) and Vector Boson Fusion (VBF) production mechanisms. While, in the former case, the vector resonance is produced via the annihilation of the quarks of the colliding protons, in the latter, the resonance is created via the fusion of the electroweak vector bosons emitted by the colliding protons.

In our calculations, $\sigma(p p \rightarrow a b X)$ is approximated by the sum of the DY and VBF cross sections. ${ }^{3}$ We ignore the top quark involvement in the vector resonance production and approximate the CKM matrix by the unity. On the other hand, we demonstrate in this section that the bottom quark contribution to the production cannot be neglected. Therefore, the DY production of our triplet resonance can proceed only via $u \bar{u}, d \bar{d}, c \bar{c}, s \bar{s}, b \bar{b} \rightarrow \rho^{0}$, and $u \bar{d}, c \bar{s} \rightarrow \rho^{+}$(+c.c.).

In the VBF production, the vector resonance can emerge from $W^{+} W^{-} \rightarrow \rho^{0}$ and $W^{+} Z \rightarrow \rho^{+}$(+c.c.). This production is calculated in the Effective-W Approximation (EWA) [61] considering the longitudinal W/Z degrees of freedom only.

\subsection{Production cross section}

The first factor in the calculation of the cross section (27) for the given decay channel is the production cross section $\sigma_{\text {prod }}(p p \rightarrow \rho X)$. We address its calculation in this Subsection. The production cross section of a resonance can be expressed as

$\sigma_{\text {prod }}(p p \rightarrow \rho X)=\left.\sum_{A \leq B} 16 \pi^{2} K_{A B} F_{A B} \frac{d \Pi_{A B}}{d \hat{s}}\right|_{\hat{s}=M_{\rho}^{2}}$

where $F_{A B}=\Gamma_{\rho \rightarrow A B} / M_{\rho}$ and $\Gamma_{\rho \rightarrow A B}$ are, respectively, the partial fatness and the partial decay width of the resonance to the partons $A$ and $B$ of the colliding protons. Furthermore, $d \Pi_{A B} / d \hat{s}$ is the differential luminosity of the colliding partons, and

$K_{A B}=\frac{2 J+1}{\left(2 S_{A}+1\right)\left(2 S_{B}+1\right)} \frac{C}{C_{A} C_{B}}$,

\footnotetext{
3 The gluon-gluon production via loops is excluded by the LandauYang theorem.
} 
where $J$ is the spin of the resonance, $C$ is its color factor, and $S_{A}, S_{B}$ and $C_{A}, C_{B}$ are the spins and colors of the initial partons, respectively. ${ }^{4}$ In this paragraph, by "partons" we also refer to the electroweak gauge bosons emitted of the partonic quarks of the colliding protons in the case of the VBF production.

Note that the model dependence can enter the production cross section (28) only via the partial decay widths $\Gamma_{\rho \rightarrow A B}$. The concerned widths include $\Gamma_{u u}, \Gamma_{d d}, \Gamma_{c c}, \Gamma_{s s}$, $\Gamma_{u d}, \Gamma_{c s}, \Gamma_{b b}, \Gamma_{W W}$ and $\Gamma_{W Z}$ where $\Gamma_{u u}=\Gamma_{c c}, \Gamma_{d d}=\Gamma_{s s}$, $\Gamma_{u d}=\Gamma_{c s}$. In the VBF production processes, all $F_{A B}$ 's are proportional to $M_{\rho}^{4} / g^{\prime \prime 2}$ up to some small corrections of higher order. All DY partial fatnesses $F_{A B}$, but the $F_{b b}$ one, are proportional to $1 / g^{\prime \prime 2}$ and do not depend on $M_{\rho}$. The $b b$ channel is the only one through which the production cross section is sensitive to the couplings of the direct interactions. If the direct couplings are sufficiently large then $F_{b b}$ is virtually proportional to $g^{\prime \prime 2}\left(b_{L}^{2}+p^{4} b_{R}^{2}\right)$. Neither this fatness depends on $M_{\rho}$.

The production cross section (28) also depends on the parton contents of the proton via the differential parton luminosities $d \Pi_{A B} / d \hat{s}$, or "quasi-luminosities" for short. In the DY process, the quasi-luminosities for various partons are obtained from the standard formula

$$
\begin{aligned}
\frac{d \Pi_{A B}}{d \hat{s}}= & \frac{1}{s} \int_{\tau}^{1} \frac{d x}{x} \frac{1}{1+\delta_{A B}} \\
& \times\left[f_{A}(x, \hat{s}) f_{B}(\tau / x, \hat{s})+A \leftrightarrow B\right],
\end{aligned}
$$

where $s$ and $\hat{s}$ are the squared center of mass energies of the colliding protons and quarks, respectively, $f_{A}(x)$ is a p.d.f. of the quark $A$ with the momentum fraction $x$ of its proton's momentum, and $\tau=\hat{s} / s$. The formula for the quasiluminosity in the VBF production reads

$$
\begin{aligned}
\frac{d \Pi_{A B}}{d \tau}= & \sum_{i \leq j} \frac{1}{1+\delta_{i j}} \int_{\tau}^{1} \frac{d x_{1}}{x_{1}} \int_{\tau / x_{1}}^{1} \frac{d x_{2}}{x_{2}} \\
& \times\left[f_{i}\left(x_{1}, q^{2}\right) f_{j}\left(x_{2}, q^{2}\right) \frac{d L_{A[i] B[j]}}{d \hat{\tau}}+i \leftrightarrow j\right],
\end{aligned}
$$

where $\hat{\tau}=\tau /\left(x_{1} x_{2}\right)$, and $d L_{A[i] B[j]} / d \hat{\tau}$ is the luminosity for two vector bosons $A$ and $B$ emitted from $i$ th and $j$ th quarks, respectively.

The vector boson luminosity $d L_{A[i] B[j]} / d \hat{\tau}$ is calculated using the EWA method. This approach is also a subject to some assumptions and restrictions. First of all, the gauge bosons are assumed to be emitted on-shell and in small angles to their parental quarks. Secondly, the masses of the fusing gauge bosons should be much smaller than the produced res-

${ }^{4}$ The tBESS vector resonance values: $J_{\rho}=1, C_{\rho}=1, S_{q}=1 / 2$, $C_{q}=3, J_{W_{L}, Z_{L}}=0, C_{W_{L}, Z_{L}}=1$. Consequently, $K_{q q^{\prime}}=1 / 12$, $K_{W_{L} W_{L}}=K_{W_{L} Z_{L}}=3$.

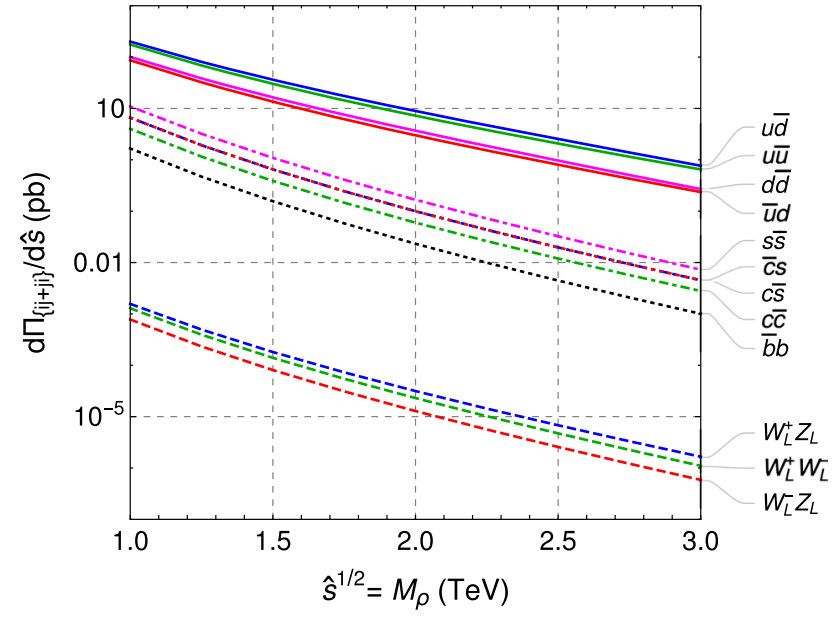

Fig. 6 Quasi-luminosities for the DY and VBF LHC processes at $\sqrt{s}=$ $13 \mathrm{TeV}$ as functions of the CMS energy of the colliding partons

onance mass. Finally, the transversal and longitudinal polarizations of the emitted gauge bosons are to be considered as separate modes.

In the presence of the deviations from the SM, the longitudinal mode usually dominates. Therefore, in our calculations, we consider contributions from this mode only. The EWA luminosity for two longitudinal vector bosons $A$ and $B$ emitted from $i$ th and $j$ th quarks reads

$$
\begin{aligned}
\frac{d L_{A[i]_{L} B[j]_{L}}}{d \hat{\tau}}= & \frac{v_{A[i]}^{2}+a_{A[i]}^{2}}{4 \pi^{2}} \frac{v_{B[j]}^{2}+a_{B[j]}^{2}}{4 \pi^{2}} \\
& \times \frac{1}{\hat{\tau}}[(1+\hat{\tau}) \log (1 / \hat{\tau})-2(1-\hat{\tau})],
\end{aligned}
$$

where $v_{A[i]}$ and $a_{A[i]}$ are the vector and axial-vector couplings of the electroweak gauge boson $A$ to the quark current of $q_{i}$, respectively.

In Fig. 6, the quasi-luminosities for the DY and VBF LHC processes at $\sqrt{s}=13 \mathrm{TeV}$ are shown. For the numerical evaluation the Mathematica package ManeParse [62] with the CT10 p.d.f. set from the LHAPDF 6 library in the HepForge repository [63] is used. As can be seen, the DY quasiluminosities dominate by many orders of magnitude over the quasi-luminosities of the VBF production. It is because the VBF production process is suppressed against the DY production process by two orders in the perturbative expansion.

The relative sizes of the individual DY quasi-luminosities can be understood in terms of the proton parton contents for individual flavors. The valence quark quasi-luminosities clearly stand over the sea quark ones. The $b b$ quasiluminosity is the smallest one by $2-3$ orders of magnitude below the $u$ and $d$ related quasi-luminosities. This poses a question whether the contribution of the vector resonance production via the sea quark annihilation are worthy of consideration. Of these, the $b \bar{b} \rightarrow \rho^{0}$ production is the most disputable. On the one hand, the $b \bar{b}$ quasi-luminosity con- 


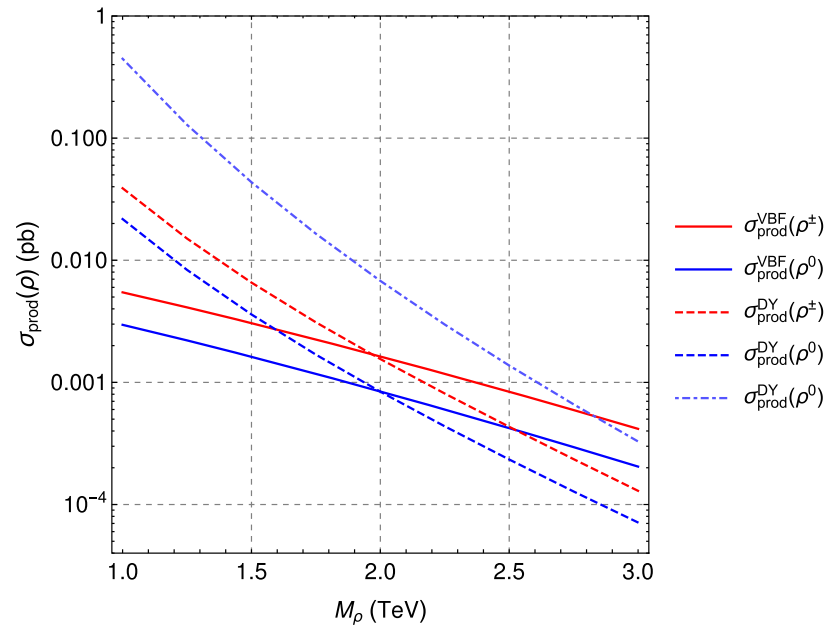

Fig. 7 The production cross sections $\sigma_{\text {prod }}(p p \rightarrow \rho X)$ for the DY and VBF productions of the neutral (blue) and charged (red) vector resonances as functions of the resonance mass when $g^{\prime \prime}=20$. The solid lines stand for the VBF production which is insensitive to the direct interactions. The dashed lines denote the DY production without the direct interactions. The dot-dashed line shows the neutral DY case with the direct interactions, namely $b_{L}=b_{R}=0.1, p=1$

tributes the least. On the other hand, this is the only production process that is sensitive to the direct interactions.

To address this issue the production cross section (28) is plotted as a function of the resonance mass for the DY and VBF modes assuming $g^{\prime \prime}=20$. In the DY case, we also distinguish between the situations with and without the direct interactions. However, only the neutral resonance DY production is sensitive to the direct interactions. Therefore, turning the direct interactions on by setting $b_{L}=b_{R}=0.1$, $p=1$ affects only the neutral DY mode. All these plots are shown in Fig. 7. The plotted production cross sections are calculated considering contributions of all quark flavors but the top quark to the proton partonic contents.

The following observations can be made in Fig. 7. First of all, in spite of much smaller quasi-luminosity, the VBF production cross section is comparable with the DY one. It is because the VBF production quasi-luminosity handicap is counter-balanced with the dominance of $F_{W W, W Z}$ over $F_{q q}$ (see Figs. 2, 3). Therefore, both production modes must be considered in our analysis. The different slopes of the DY and VBF curves can be understood when we recall that $F_{q q} \propto M_{\rho}^{0}$ while $F_{W W, W Z} \propto M_{\rho}^{4}$.

Secondly, we can see that the direct interactions can have a visible impact on the production cross section for the neutral DY mode. When $M_{\rho}=1 \mathrm{TeV}$ and $g^{\prime \prime}=20$ the contributions of $b \bar{b} \rightarrow \rho^{0}$ to the production cross section is $12 \%$ and $95 \%$ when $b_{L}=b_{R}=0.01, p=1$ and $b_{L}=b_{R}=0.1, p=1$, respectively.

Since the sea-without- $b$ quark production of the vector triplet is not sensitive to the direct interactions, it is reason- able to expect that its contribution to the production cross section will be small under all circumstances. The size of this contribution cannot be read off of Fig. 7. In our calculations, we have established that by ignoring the sea quark production in the no-direct-interaction situation the production cross section is lowered by about $7 \%$ when $M_{\rho}=1 \mathrm{TeV}$ and by about $5 \%$ at $M_{\rho}=3 \mathrm{TeV}$. The discrepancy decreases as $M_{\rho}$ grows.

\section{$3.2 \sigma_{\text {prod }} \times \mathrm{BR}$}

Following Eq. (27), $\sigma(p p \rightarrow \rho X \rightarrow a b X)$ is obtained when the production cross section is multiplied by the branching ratio of $\rho \rightarrow a b$. When there are no direct interactions of the vector triplet with top and bottom quarks more than $99 \%$ of $\rho$ decays into the $W W / W Z$ channel. It can be seen in Fig. 2 . To a high accuracy, this assertion holds for any considered values of $g^{\prime \prime}$ and $M_{\rho}$. Therefore, $\sigma_{\text {prod }} \times \operatorname{BR}(\rho \rightarrow W W / W Z)$ is virtually identical with the production cross section $\sigma_{\text {prod }}$.

The direct interactions influence the resulting cross sections solely via the branching ratios with a single exception. The exception is the neutral resonance production via the DY mode. There, the production depends on the parameters of the direct interactions as well. Recall that the dependence originate in the $b \bar{b} \rightarrow \rho^{0}$ vertex.

The direct interactions can alter the vector resonance branching ratios of the individual channels significantly. It can be inferred from the behavior of $\operatorname{BR}(\rho \rightarrow W W / W Z)$ and $\operatorname{BR}(\rho \rightarrow t t / b b / t b)$ as it is depicted in Figs. 3, 4, and 5. The prevailing behavior of these branching ratios is that $\sigma_{\text {prod }} \times \operatorname{BR}(\rho \rightarrow t t / b b / t b)$ grows with the strength of the direct interactions while $\sigma_{\text {prod }} \times \operatorname{BR}(\rho \rightarrow W W / W Z)$ decreases. Nevertheless, there are particular combinations of the values of $b_{L, R}$ and $p$ when this statement does not hold. For example, if $b_{L}=0$ and $p=0$ the vector resonance decays strongly to $t t$ while it decays to $b b$ only via the indirect interactions not sensitive to these parameters. In such a case, $\operatorname{BR}(\rho \rightarrow t t)$ grows with $b_{R}$ while $\operatorname{BR}(\rho \rightarrow b b)$ decreases. This very behavior will transfer without alteration into the cross sections of the processes where the neutral resonance is not DY produced. Otherwise, the cross section dependence on the direct interaction parameters will result from the interplay between the BR and production cross section dependences.

In our previous paper [39], we investigated the tBESS mass exclusion limits for the no direct interactions case. In this analysis, all sea partonic quarks, including the $b$ quarks, were ignored. It was a well-justified assumption for the case. Nevertheless, in the paper, we also commented on our expectation regarding the exclusion limits for the case with the direct interactions. Neglecting the $b$ quarks, we expected that the direct interactions would lower the cross sections for the $W W / W Z$ channels and increase the cross sections for the 
$t t, b b / t b$ channels. Since the latter channels did not reach the experimental upper limits for any admissible values of $b_{L, R}$, $p$, we predicted that the presence of the direct interactions would relax the mass exclusion limits. Now, we understand that the bottom quark contribution to the vector resonance production can be ignored only for particular selections of the direct interaction parameters. Thus, our expectation was not correct except for these particular cases.

\section{The exclusion limits on the vector resonance mass}

In this Section, we work out the mass exclusion limits for the vector triplet of our model by the confrontation of its cross sections (27) with the experimental upper bounds provided by the ATLAS and CMS Collaborations. The regions of the parameter space where the predicted cross section exceeds the upper bounds are excluded. We review fourteen vector resonance decay channels available to the date of this analysis: $W Z$ [64-67], $W W[64$,

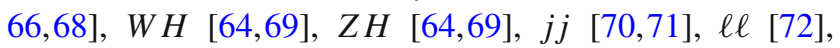
$\ell v[73], \tau \tau$ [74], $\tau \nu$ [75], $t t$ [76], bb [77], and $t b$ [78,79], where $\ell=e, \mu$. The corresponding 95\% C.L. bounds used in this Section are based on the integrated luminosity of about $36 \mathrm{fb}^{-1}$ (full 2016 data) or less. As was discussed in previous Sections, various experimental and theoretical considerations restrict our mass exclusion limit searches to the following region of the parameter space: $1 \leq M_{\rho} / \mathrm{TeV} \leq 3,12 \leq g^{\prime \prime} \leq 25\left|b_{L, R}\right| \leq 0.1$, $0 \leq p \leq 1$.

As $g^{\prime \prime}$ approaches the naive perturbative limit of 25 , the radiative corrections grow and our tree-level results become less reliable. However, various precision levels are needed for addressing various questions. Therefore, we present results for $g^{\prime \prime}$ up to the naive perturbative limit and leave up to the reader to choose the maximal trustworthy value of $g^{\prime \prime}$ for his/her purpose. In addition, while the inclusion of the radiative corrections would alter the obtained exclusion limits it does not invalidate the qualitative lessons learned from our analysis.

After the analysis advertised above has been finalized new experimental upper bounds have been published by the ATLAS and CMS Collaborations. Some of them were still based on the $36 \mathrm{fb}^{-1}[80,81]$ dataset, other stemmed from the bigger 77-80 $\mathrm{fb}^{-1}$ [82,83] and $139 \mathrm{fb}^{-1}$ [84-86] datasets. At the end of this Section we append an update on how these new upper bound affect our mass exclusion limits. Nevertheless, since the analysis of all collected LHC data by the Collaborations is still in progress, even these updates of the mass limits can become obsolete in the near future. Yet, we believe that the presented work provide valuable experience independent of the actual values of the mass exclusion limits.

\subsection{No direct interactions}

The no direct interactions case was already analyzed in our previous work [39]. Out of all inspected channels there, only the $W W$ and $W Z$ channels provided the exclusion limits for the vector resonance mass.

In this paper, the mass exclusion limits based on the updated upper bounds are presented. Besides the channels considered in [39], a new decay channel, the $\tau \nu$ one [81], has also been added to the current study. The distinction of the DY and VBF production modes for the $W W / W Z$ upper bounds is another novelty. Finally, the proton partonic contents includes the $s, c$, and $b$ quarks in the calculation of the model's cross section. All these updates have not altered the conclusion of the previous paper [39] that the $W W$ and $W Z$ channels are the only channels providing the mass exclusion limits for the tBESS vector triplet with no direct interactions. Of course, the limits themselves have been changed by this analysis upgrade.

Taking into account the separate upper bounds for both production modes of the $W W$ and $W Z$ channels, our exclusion limits are based on the following six processes: (a) the DY production of $W W$ and $W Z$, (b) the VBF production ${ }^{5}$ of $W W$ and $W Z$, and (c) the DY+VBF production of $W W$ and $W Z$. In Fig. 8, the tBESS cross sections for all these modes as functions of $M_{\rho}$ are shown at $g^{\prime \prime}=12,16,20$, and 24 . In addition, the applicable experimental upper bounds from the six channels mentioned above are superimposed on the graphs. The mass regions where the predicted cross section exceeds the experimental upper bound are experimentally excluded.

To avoid the cluttering of the Fig. 8 graphs with unnecessary curves, only the most restricting experimental upper bounds are displayed there. In the $W Z_{\mathrm{DY}}, W W_{\mathrm{DY}}$, and $W W_{\mathrm{VBF}}$ graphs, the most restricting bounds are provided by a single curve. In the remaining cases, the most restrictive upper bounds are comprised of two curves, each providing the most restrictive bound for a different subregion of the considered resonance mass interval of 1-3 TeV. The sources of the displayed upper bound curves of the individual channels are summarized in Table 1 . The table also indicates the particular final states that were used to obtain the bounds.

In the tBESS model, the neutral and charged vector resonances are virtually degenerate in mass. Therefore, the exclusion limit is obtained as the higher one of the charge and neutral limits. In particular, we take the most stringent of the limits found in all six processes displayed in Fig. 8. Of course, we can also establish the exclusion limits for individual charge modes independently of each other. The lessons

\footnotetext{
5 The sensitivity of the LHC vector boson scattering processes, $W Z \rightarrow$ $W Z$ and $W W \rightarrow W W$, to new vector resonances was also investigated recently in $[87,88]$.
} 

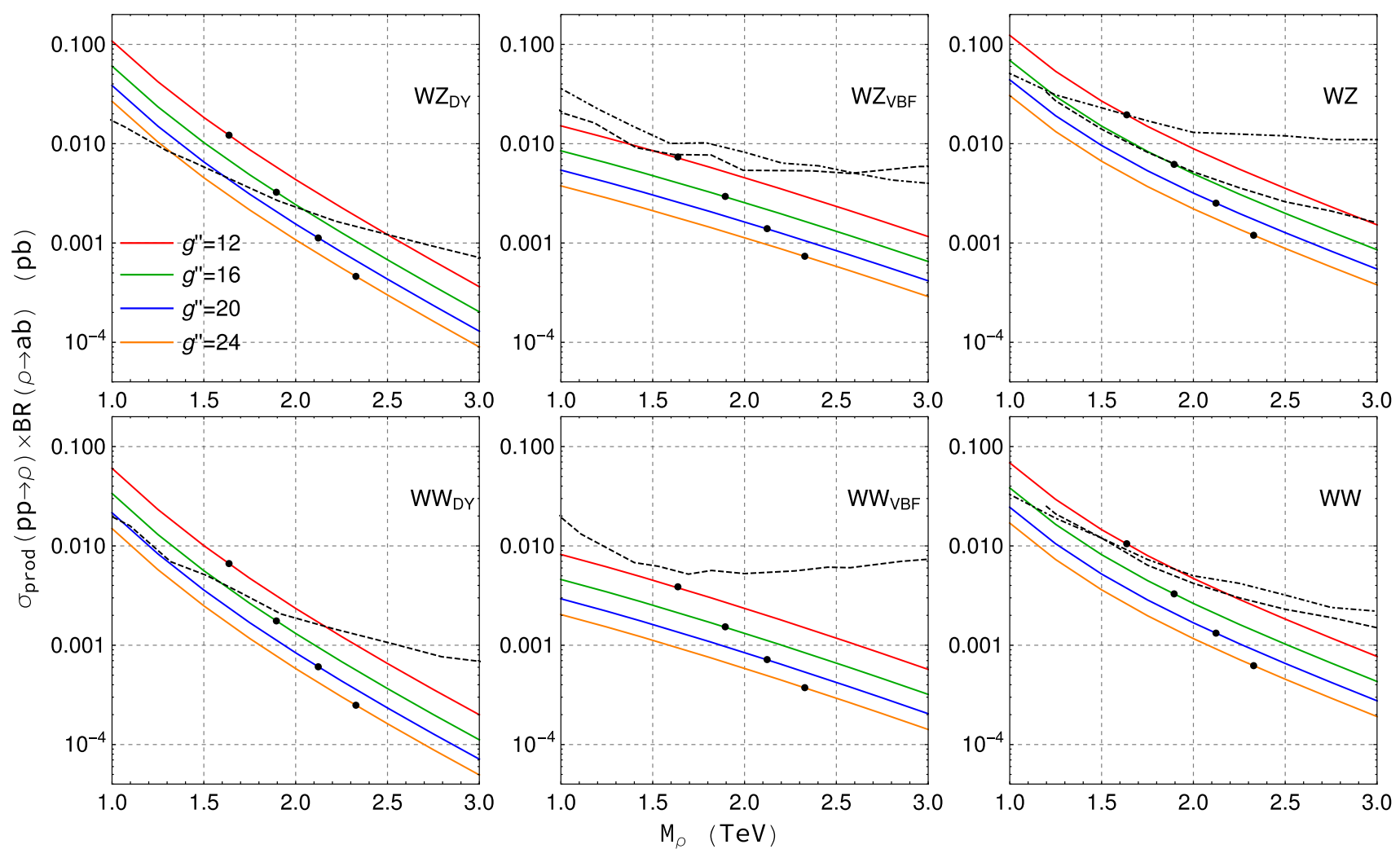

Fig. 8 The plots of $\sigma_{\text {prod }} \times$ BR predicted by the tBESS model for the $W W$ and $W Z$ channels (solid lines) along with the relevant experimental upper bounds (dashed and dot-dashed lines) as functions of $M_{\rho}$ assuming no direct interactions of the vector triplet with the top and bottom quarks. The predicted cross sections are shown for four different values of $g^{\prime \prime}: 12$ (red), 16 (green), 20 (blue), 24 (orange). They decrease with $g^{\prime \prime}$. The black dots indicate the resonance mass at which the resonance fatness amounts to $10 \%$. The first row of the graphs correspond to the $W Z$ final state, the second one to the $W W$ final state. The first, second and third columns of the graphs correspond to the DY production, the VBF production and the combination of both, respectively. In all graphs, the $13 \mathrm{TeV}$ proton-proton collisions are considered
Table 1 The sources of the experimental upper bounds for the $W W$ and $W Z$ channels depicted in Fig. 8. Beside the decay channel, the first column also indicates whether the considered production proceeds via the DY production, the VBF production or both. The second column shows what is the integrated luminosity of the data sample used to derive the bound. The third column indicates the final states through which the given channel was observed. The bounds were provided by the ATLAS and CMS Collaborations in the papers referred to in the last column

\begin{tabular}{llll}
\hline Channel & Luminosity $\mathrm{fb}^{-1}$ & Final state & References \\
\hline$W Z_{\mathrm{DY}}$ & 36.1 & $q q q q+v v q q+\ell v q q+\ell \ell q q+\ell \ell \ell v$ & {$[64]$} \\
$W Z_{\mathrm{VBF}}$ & 36.1 & $v v q q+\ell v q q+\ell \ell q q+\ell \ell \ell v$ & {$[64]$} \\
$W Z$ & 36.1 & $\ell \ell q q+v v q q$ & {$[65]$} \\
& 35.9 & $j j$ & {$[67]$} \\
$W W_{D Y}$ & 13.2 & $\ell \ell q q$ & {$[64]$} \\
$W W_{V B F}$ & 36.1 & $q q q q+\ell v q q+\ell v \ell v$ & {$[64]$} \\
$W W$ & 36.1 & $\ell v q q+\ell v \ell v$ & {$[66]$} \\
& 35.9 & $j j$ & {$[68]$} \\
\hline
\end{tabular}

learned from the tBESS vector triplet model can be applied to other models with similar phenomenological traits.

The tBESS exclusion mass limits for different values of $g^{\prime \prime}$ within $14 \leq g^{\prime \prime} \leq 25$ are listed in Table 2 . The exclusion limits for $g^{\prime \prime}$ values below 14 are not shown because their fatness exceeds $40 \%$ which makes the limits obtained via the NWA calculations unreliable. 
Table 2 The mass exclusion limits (MEL) for the tBESS vector resonance triplet without direct interactions for various values of $g^{\prime \prime}$. The second row contains the values of the resonance fatness. All MEL values shown in the table indicate the upper boundaries of the mass exclusion region

\begin{tabular}{lllllllllllll}
\hline$g^{\prime \prime}$ & 14 & 15 & 16 & 17 & 18 & 19 & 20 & 21 & 22 & 23 & 24 & 25 \\
\hline$\Gamma_{\text {tot }} / M_{\rho}$ & 0.39 & 0.21 & 0.14 & 0.10 & 0.07 & 0.05 & 0.03 & 0.02 & 0.02 & 0.01 & 0.01 & $<0.01$ \\
$\operatorname{MEL}(\mathrm{TeV})$ & 2.50 & 2.21 & 2.07 & 1.95 & 1.83 & 1.70 & 1.60 & 1.51 & 1.43 & 1.37 & 1.31 & 1.24 \\
\hline
\end{tabular}

\subsection{The direct interactions included}

In this paper, the impact of the direct interactions on the tBESS vector triplet mass exclusion limits is studied for the first time. The direct interactions can affect the limits by increasing the cross sections of the top and bottom quark decay channel processes. It remains to be seen if the increase is sufficient for the cross sections to reach the experimental upper bounds, in some regions of the parameter space at least. Certainly, the direct interactions also affect the cross sections of the $W$ and $Z$ decay channel processes. Thus, the mass exclusion limits will be influenced by the direct interactions even if the top and bottom quark processes fail in providing additional restrictions.

The current upper bounds based on the data from the remaining channels-with leptons, light quarks, and the Higgs boson-are too weak to modify the tBESS triplet mass exclusion limits. Even though the introduction of the direct couplings does affect the tBESS cross sections of these channels, their values remain far below their upper bounds. They also cannot compete with the $t t / b b / t b$ cross sections once the strength of the direct interactions exceeds the level of $\left|b_{L, R}\right| \geq 0.02-0.03$. Therefore these channels are not able to contribute to the tBESS mass exclusion limits at the current amounts of the collected data. We are not going to further discuss these channels in this paper.

\section{$t b, t t, b b$}

The $t b$ channel cross section does not provide the mass exclusion limits for any considered values of the model parameters. In this channel, the sensitivity to the direct interactions enters via the $\operatorname{BR}(\rho \rightarrow t b)$. With the growing strength of the direct interactions, the branching ratio also grows, reaching the limiting value of 1 as $\left(b_{L}^{2}+p b_{R}^{2}\right) \rightarrow \infty$. Note, however, that there are regions of the parameter space where $\operatorname{BR}(\rho \rightarrow t b)$ exceeds $90 \%$ already at $\left|b_{L, R}\right|=0.1$ (see Figs. 3, 5). In principle, $\sigma_{\text {prod }} \times \mathrm{BR}(\rho \rightarrow t b)$ can assume any value between the no direct interaction cross section and the production cross section if the suppressing Death Valley region of the parameter space is ignored. In Fig. 9, the plots of these two extremes of the tBESS cross sections as functions of $M_{\rho}$ for various $g^{\prime \prime}$ are shown along with the most restricting experimental upper bounds for this channel. It demonstrates that the current experimental bounds do not exclude the production cross

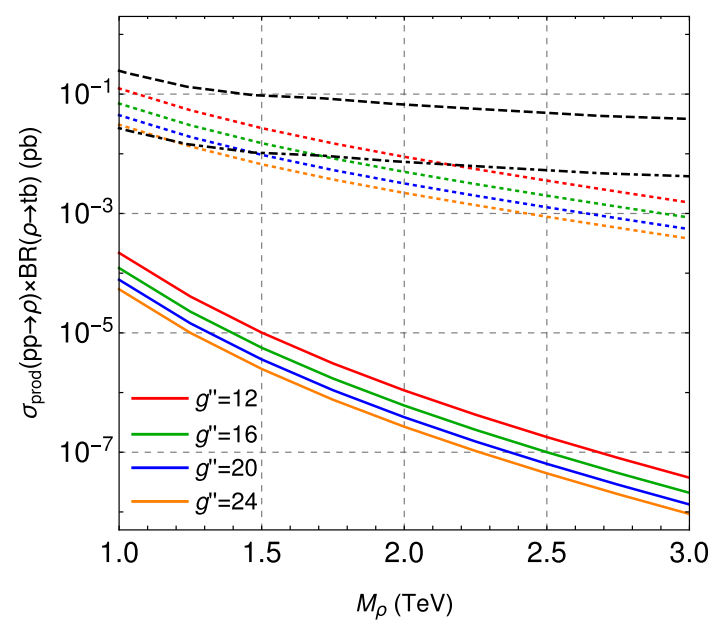

Fig. 9 The plots of $\sigma_{\text {prod }} \times$ BR predicted by the tBESS model for the $t b$ channel when there are no direct interactions (colored solid lines) and when $\operatorname{BR}(\rho \rightarrow t b)=1$ (colored dotted lines) along with the relevant experimental upper bounds (black dashed line) as functions of $M_{\rho}$. The black dot-dashed line depicts the expected upper bound for the integrated luminosity $3000 \mathrm{fb}^{-1}$. The predicted cross sections are shown for four different values of $g^{\prime \prime}: 12$ (red), 16 (green), 20 (blue), 24 (orange). They decrease with $g^{\prime \prime}$. The $13 \mathrm{TeV}$ collisions of protons are assumed

section values. In addition, the estimated upper bound, when the integrated luminosity reaches $3000 \mathrm{fb}^{-1}$ (HL-LHC), is also plotted in the graph. ${ }^{6}$ It seems that the $3000 \mathrm{fb}^{-1}$ luminosity will be needed to restrict the vector resonance mass from the data in this channel.

As far as the $t t$ and $b b$ channels are concerned, they are sensitive to the direct interactions through the $b \bar{b} \rightarrow \rho^{0}$ production, as well as the vector resonance decays to $t \bar{t}$ and $b \bar{b}$. Nevertheless, in these channels, no mass exclusion limits for the parameter space region under consideration are implied by the current experimental data. The list of the currently most restricting upper bounds, this conclusion is based upon, is shown in Table 3.

$W W, W Z$

In the no direct interaction case, the $W W$ and $W Z$ channels provided the only mass exclusion limits for the tBESS vector triplet. Once the direct interactions are introduced, the pre-

6 The hypothetical HL-LHC bound is obtained by a simple rescaling of the current $35.9 \mathrm{fb}^{-1}$ bound by the factor of $\sqrt{35.9 / 3000}$. 
Table 3 The sources of the experimental upper bounds for the $t t, b b$, and $t b$ channels we use in our analysis

\begin{tabular}{llll}
\hline Channel & Luminosity & Final state & References \\
\hline$t t$ & $35.9 \mathrm{fb}^{-1}$ & Dileptons + lepton + hadronic & {$[76]$} \\
$b b$ & $36.1 \mathrm{fb}^{-1}$ & 2- $b$ jets & {$[77]$} \\
$t b$ & $36.1 \mathrm{fb}^{-1}$ & Semileptonic + hadronic & {$[78]$}
\end{tabular}

dicted cross section gets modified and become dependent on the values of $b_{L, R}$ and $p$. The $W Z_{\mathrm{DY}}, W Z_{\mathrm{VBF}}, W Z_{\mathrm{DY}+\mathrm{VBF}}$, and $W W_{\mathrm{VBF}}$ cross sections diminish in comparison with the no direct interaction case for all parameter values under consideration. It is because these cross sections depend on $b_{L, R}$ and $p$ through $\operatorname{BR}(\rho \rightarrow W W)$ and $\operatorname{BR}(\rho \rightarrow W Z)$ only.

The behavior of the $W W_{\mathrm{DY}}$ cross section is not so easy to conjecture. Its sensitivity to the direct interactions originates not only in $\operatorname{BR}(\rho \rightarrow W W)$ but also in the production of $\rho$ through the $b \bar{b}$ annihilation. Of course, this feature impacts the exclusion limits obtained from the combined (DY+VBF) $W W$ cross section.

When $\left|b_{L, R}\right| \leq 0.1$ the cross sections for all three $W Z$ modes lie in the bands between the no direct interaction cross section (the upper boundary) and the $b_{L}=-b_{R}=-0.1$ and $p=1$ cross section (the lower boundary). ${ }^{7}$ The upper boundary of the cross section stripe for the $W W_{\mathrm{VBF}}$ mode is also determined by the no direct interaction cross section. The lower boundary is given by the cross section at $b_{L}=$ $b_{R}=-0.1$ and $p=1$.

Regarding the $W W_{\mathrm{DY}}$ and $W W_{\mathrm{DY}}+\mathrm{VBF}$ modes, their cross sections can either grow or decrease with the growing strength of the direct interactions, depending on the particular combination of the $\left(b_{L, R}, p\right)$ parameter values. This more complex behavior originates from the competition between the growing production cross section and the shrinking branching ratio. We determined numerically that when $\left|b_{L, R}\right| \leq 0.1$ the $W W_{\mathrm{DY}}$ and $W W_{\mathrm{DY}}+\mathrm{VBF}$ cross sections are bound from below by the cross section for $b_{L}=0, b_{R}=-0.1, p=0$. From above, they are bound by the $b_{L}=-b_{R}=-0.1$ and $p=1$ cross section. This behavior is illustrated in Fig. 10 where the bands of possible values of the $W W$ and $W Z$ cross sections as functions of $M_{\rho}$, when $\left|b_{L, R}\right| \leq 0.1$ and $0 \leq p \leq 1$, are depicted. The bands are constructed for two values of $g^{\prime \prime}$, namely 16 and 20. In addition, the same experimental upper bounds, as in Fig. 8, are superimposed. We can see that the $W W_{\mathrm{VBF}}$ and $W Z_{\mathrm{VBF}}$ provide no mass exclusion limits for the vector triplet of our model, while the remaining modes do.

Since there are too many free parameters involved, and the behavior of some modes is not simple, it is not possible to find a way to present the mass exclusion limits in a

\footnotetext{
7 The $W Z_{\mathrm{DY}}, \mathrm{VBF}, \mathrm{DY}+\mathrm{VBF}$ and $W W_{\mathrm{VBF}}$ cross sections for all combinations of $b_{L, R}$ values such that $\left|b_{L, R}\right|=0.1$ and $p=1$ are virtually identical.
}

concise manner. Nevertheless, the graphs in Fig. 10 provide information about certain aspects of the mass exclusion limit behavior: whether there are any limits at all, what is the range of their possible values, and so on.

If additional restrictions on the free parameters were imposed more specific information about the exclusion limits could be obtained. For example, let us reduce the number of free parameters of the tBESS model by assuming that $b_{L}=b_{R}$ and $p=1$. It means that the direct interactions are parameterized by a single free parameter $b \equiv b_{L}=b_{R}$. In Table 4 , the smallest mass exclusion limits that can be found from the current $W W$ and $W Z$ experimental upper bounds for various values of $g^{\prime \prime}$ when $|b| \leq 0.1$ are shown. The table also displays the value of $b$ and the value of the resonance fatness $\Gamma_{\text {tot }} / M_{\rho}$ that corresponds to the found mass exclusion limit.

The second reduction of the tBESS model we analyze here is defined by $b_{L}=0$ and $b_{R}=0.1$. That describes the situation when there are no direct interactions of the vector resonance to the left top and left bottom quarks. The interaction with the right top quark is at the maximum considered in this paper and the interaction with the right bottom quark can be weakened by the only remaining free parameter $p$. In Table 5, we show the smallest mass exclusion limits that can be found from the current $W W / W Z$ experimental upper bounds for various values of $g^{\prime \prime}$ when $0 \leq p \leq 1$. In the table, we also show the value of $p$ and the value of the resonance fatness $\Gamma_{\text {tot }} / M_{\rho}$ that correspond to the found mass exclusion limit. Note that the used experimental upper bounds provide no mass exclusion limits for $g^{\prime \prime} \geq 21$.

To achieve a better understanding of this complex multiparameter situation we can plot the regions of the $\left(b_{L}, b_{R}\right)$ parameter subspace for which a certain resonance mass is excluded by the experimental upper bounds when the values of $g^{\prime \prime}$ and $p$ are also fixed. As an example, we choose $M_{\rho}=$ $1.8 \mathrm{TeV}, g^{\prime \prime}=18$ and $p=0.8$. With this choice of the parameters the resonance fatness amounts to $\Gamma_{\text {tot }} / M_{\rho}=6 \%$ at $b_{L}=b_{R}=0$. As both, $\left|b_{L}\right|$ and $\left|b_{R}\right|$ approach 0.1 the fatness grows to $9 \%$. Thus we can expect that the deviations introduced by the NWA calculations are reasonably small. The resulting plot of the experimentally excluded regions in the $\left(b_{L}, b_{R}\right)$ space is shown in Fig. 11. The excluded region is obtained by the union of the areas excluded by the $W Z_{\mathrm{DY}}$ and $W W_{\text {DY }}$ decay channels. 

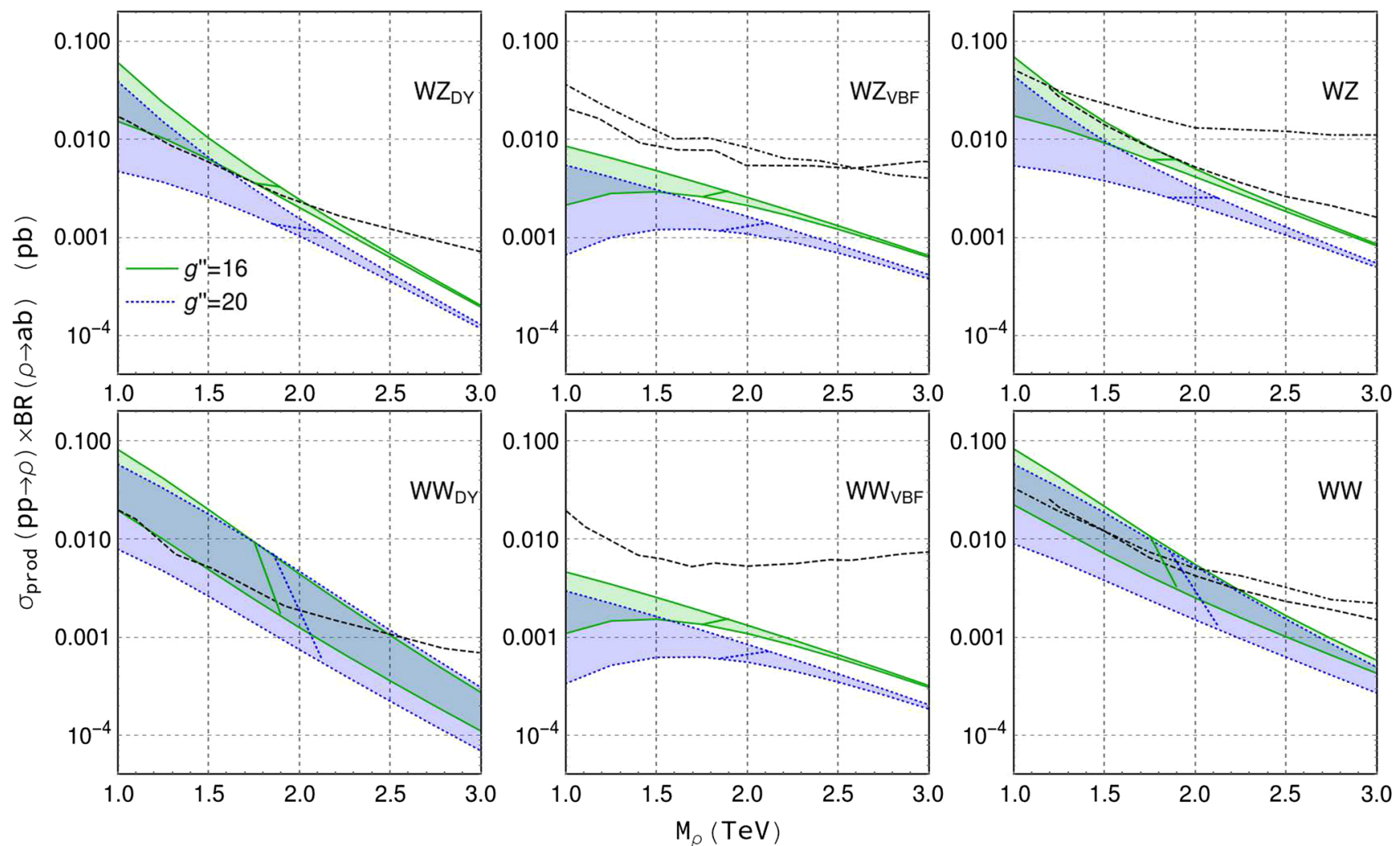

Fig. 10 The bands of possible values of $\sigma_{\text {prod }} \times$ BR predicted by the tBESS model for the $W W$ and $W Z$ channels produced via the DY, $\mathrm{VBF}$ and $\mathrm{DY}+\mathrm{VBF}$ production modes. The bands are constructed for $g^{\prime \prime}=16$ (solid green) and $g^{\prime \prime}=20$ (dotted blue) when the direct interaction parameters are restricted to $\left|b_{L, R}\right| \leq 0.1$ and $0 \leq p \leq 1$. The bands are crossed by the lines of the same style as the bands' boundaries indicating the resonance mass at which the resonance fatness amounts to $10 \%$. The same experimental upper bounds (dashed and dot-dashed black lines) as in Fig. 8 are also shown. In all graphs, the $13 \mathrm{TeV}$ protonproton collisions are considered
Table 4 The smallest tBESS resonance mass exclusion limits (MEL) within the interval $\left|b_{L=R}\right| \leq 1$ assuming $b_{L=R} \equiv b_{L}=b_{R}$ and $p=1$ for various values of $g^{\prime \prime}$. The second and third rows contain, respec- tively, the values of $b_{L=R}$ and of the resonance fatness $\Gamma_{\text {tot }} / M_{\rho}$ that correspond to the quoted mass exclusion limit. The excluded masses lie below MEL

\begin{tabular}{|c|c|c|c|c|c|c|c|c|c|c|c|c|}
\hline$g^{\prime \prime}$ & 14 & 15 & 16 & 17 & 18 & 19 & 20 & 21 & 22 & 23 & 24 & 25 \\
\hline$b_{L=R} \times 10^{2}$ & 9.5 & 5.6 & 4.4 & 3.6 & 3.2 & 2.8 & 2.5 & 2.0 & 1.7 & 1.7 & 1.7 & 1.6 \\
\hline$\Gamma_{\text {tot }} / M_{\rho}$ & 0.36 & 0.20 & 0.14 & 0.10 & 0.06 & 0.04 & 0.03 & 0.02 & 0.02 & 0.01 & $\leq 0.01$ & $\leq 0.01$ \\
\hline MEL $(\mathrm{TeV})$ & 2.42 & 2.15 & 2.04 & 1.92 & 1.77 & 1.64 & 1.53 & 1.44 & 1.38 & 1.30 & 1.11 & 1.03 \\
\hline
\end{tabular}

Table 5 The smallest tBESS resonance mass exclusion limits (MEL) within the interval $0 \leq p \leq 1$ assuming $b_{L}=0$ and $b_{R}=0.1$ for various values of $g^{\prime \prime}$. The second and third rows contain, respectively, the values of $p$ and of the resonance fatness that correspond to the quoted mass exclusion limit. The excluded masses lie below MEL

\begin{tabular}{lllllllll}
\hline$g^{\prime \prime}$ & 14 & 15 & 16 & 17 & 18 & 19 & 20 & $21-25$ \\
\hline$p$ & 1 & 0.871 & 0.772 & 0.707 & 0.672 & 0.630 & 0.589 & - \\
$\Gamma_{\text {tot }} / M_{\rho}$ & 0.38 & 0.20 & 0.14 & 0.09 & 0.06 & 0.04 & 0.03 \\
MEL $(\mathrm{TeV})$ & 2.46 & 2.14 & 2.02 & 1.87 & 1.68 & 1.49 & 1.33 & no MEL \\
\hline
\end{tabular}

The ring-like structure depicted in Fig. 11 can be understood from the no-direct-interaction graphs of Fig. 8 and from their response to the direct interactions being turned on.
With no direct interactions, the only channel that excludes the $1.8 \mathrm{TeV}$ vector triplet is the $W Z_{\mathrm{DY}}$ one. However, the tBESS cross section of this channel decreases with the direct 
interactions strength. When the direct interactions become sufficiently strong the predicted value dives below the experimental one and the channel ceases to exclude the resonance. On the other hand, owing to the partonic bottom quark contribution, the $W W_{\text {DY }}$ decay channel cross section grows with the direct interaction strength. When the direct interactions are turned off the resonance is not excluded by the channel. However, its predicted cross section exceeds the measured value when the direct interactions become sufficiently strong and, thus, excludes the given resonance. Since the exclusion boundary of the $W Z_{\mathrm{DY}}$ channel are closer to the origin $b_{L}=b_{R}=0$ than the exclusion boundary of the $W W_{\mathrm{DY}}$ channel a ring-like region of the $\left(b_{L}, b_{R}\right)$ parameter subspace, where the resonance is not excluded, has emerged. The resonance fatness at the $W W_{\mathrm{DY}}$ boundary is $7 \%$.

The excluded region shown in Fig. 11 is very sensitive to the values of the resonance mass. This is demonstrated in Fig. 12. The figure consists of the graphs which show the excluded regions of the $\left(b_{L}, b_{R}\right)$ subspace for the values of $M_{\rho}$ that slightly vary around the mass considered at the graph of Fig. 11. The excluded regions change significantly even within the small range $1.75 \leq M_{\rho} / \mathrm{TeV} \leq 1.85$ for all three chosen values of $p=0.4,0.7,1$. We suggest that the combination of this feature and the sufficiently large imprecision caused by any used approximation can result in a quite deceiving conclusions about the exclusion of the resonance of a given mass. Thus, the deviations introduced by the NWA calculations should be carefully scrutinized as the fatness of the resonance under consideration grows.

These findings suggest that an exhaustive study of the experimentally excluded areas of the complete tBESS parameter space would be a cumbersome task even though the tBESS phenomenological Lagrangian corresponds to a relatively simplistic LHC scenario. The real scenario of the strongly-interacting extension of the SM can introduce even more complex phenomenology of the new resonances to be discovered at the LHC. Even if this is the case the tBESS model analysis can provide valuable lessons. ${ }^{8}$ While any simplifying assumptions that would help with the detection analysis are certainly appreciated, one has to remember that this can come at the price of losing sensitivity to more subtle behavior of the studied resonances.

\subsection{Flavor physics restrictions of the direct interactions}

Since the direct interaction of the tBESS vector triplet with the third generation quarks stands out, it is a source of large flavor violation. In the LHC analysis of this paper, the flavor conserving processes dominate. Nevertheless, the tBESS resonance direct interaction can significantly contribute to the

\footnotetext{
${ }^{8}$ Recently published work [47] about the LHC signals of a model with broad composite resonances can also serve for this purpose.
}

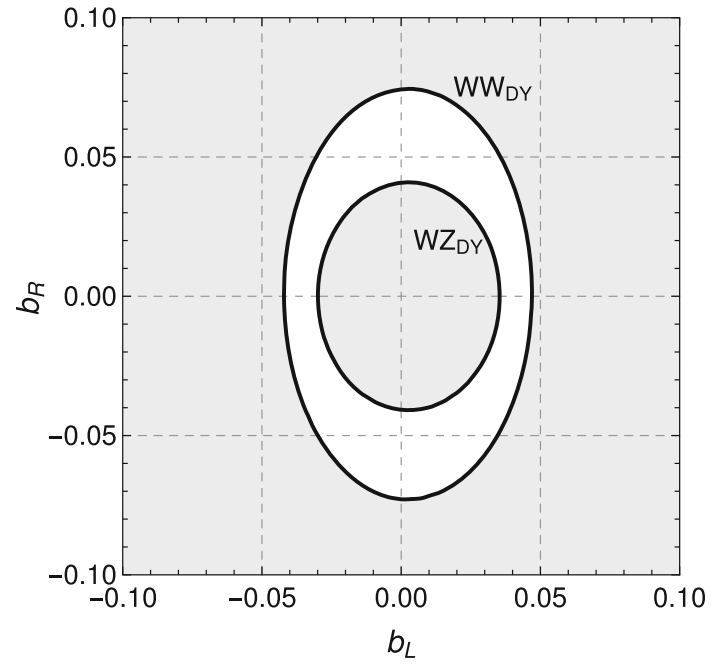

Fig. 11 The excluded region of the $\left(b_{L}, b_{R}\right)$-plane (gray area) for the vector resonance with the mass of $M=1.8 \mathrm{TeV}, g^{\prime \prime}=18, p=0.8$
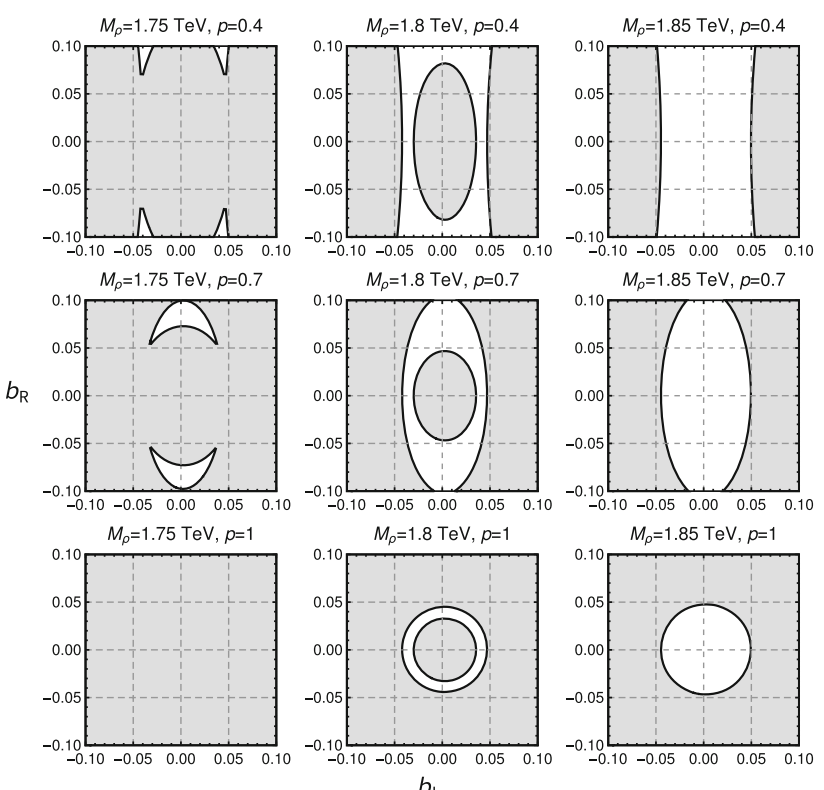

Fig. 12 The grid of graphs of the excluded regions of the $\left(b_{L}, b_{R}\right)$ plane (gray area) when $g^{\prime \prime}=18$. The mass changes from graph to graph in the left-to-right direction through the values of $1.75,1.80$, and $1.85 \mathrm{TeV}$. The $p$ parameter changes from the top to the bottom through the values of $0.4,0.7$, and 1.0

$B-\bar{B}$ mixing, the $B$-meson decays and other flavor violating phenomena. Thus, one would wonder how the flavor physics indirect limits on the $b_{L, R}$ and $p$ parameters compare with the limits derived from the LHC cross section upper bounds discussed in the previous subsection. While the exhaustive answer to this question is beyond the scope of this paper we would like to provide, at least, a partial insight using the updated versions of our previous analyses of the tBESS model. 
In the papers [36,37], we studied how flavor physics, along with other low-energy measurements, restricts the tBESS model's parameters. Among other things, the limits based on the experimental values of the $Z \rightarrow b \bar{b}$ decay width and the $B \rightarrow X_{s} \gamma$ branching ratio were derived. Rather than restricting $b_{L, R}$ and $p$ alone, the former provides limits on the $b_{L}-2 \lambda_{L}$ and $p^{2}\left(b_{R}+2 \lambda_{R}\right)$ combinations of the tBESS free parameters and the latter restricts $b_{L}-2 \lambda_{L}$ and $p \cdot\left(b_{R}+2 \lambda_{R}\right)$. Note that the free parameters $\lambda_{L, R}$ appearing in these expressions associate with the new symmetry-allowed tBESS model interaction terms of the $S U(2)_{L}$ and $U(1)_{Y}$ gauge bosons. In contrast to the $b_{L, R}$ terms, these terms do not contribute to the direct interactions of the vector triplet in the flavor basis. Nevertheless, after the mass matrix diagonalization, the physical vertices of the electroweak bosons and the tBESS vector triplet with fermions become dependent on various combinations of the $b_{L, R}, p$, and $\lambda_{L, R}$ parameters $[36,37]$. The $\lambda_{L, R}$ parameters did not appear in the text above because their impact on the analysis of the direct LHC exclusion limits is negligible. However, this is not the case for the indirect limits derived from the low-energy measurements. It was also found $[36,37]$ that these limits vary insignificantly when $g^{\prime \prime}$ changes within the $12 \leq g^{\prime \prime} \leq 25$ region considered in this paper.

The examples of the regions of the parametric space allowed by the experimental values of $\Gamma_{Z}=(2.4952 \pm$ $0.0023) \mathrm{GeV}$ and $\mathrm{BR}(Z \rightarrow b \bar{b})=(15.12 \pm 0.05) \%$ [89] for three different values of $p$ are displayed in Fig. 13. There, we can see that the allowed values of $b_{L}-2 \lambda_{L}$ and $b_{R}+2 \lambda_{R}$ are highly correlated and form a narrow strip of a circular $(p=1)$ or elliptical $(p=0.7)$ shape. As $p$ approaches zero the shape of the strip straightens up leaving no restriction on $b_{R}+2 \lambda_{R}$. The widths of the strips are about 0.015 at the $95 \%$ C.L. The regions displayed in Fig. 13 were calculated for $g^{\prime \prime}=18$. However, as mentioned before, the dependence of the obtained limits on $g^{\prime \prime}$ is weak. Roughly speaking, $b_{L}-2 \lambda_{L}$ can assume any value between -0.01 and 3.5 independently of $p$. The allowed values of $b_{R}+2 \lambda_{R}$ range between -1 and 2 when $p=1$. This interval grows as $p$ decreases.

The experimental value of $\mathrm{BR}\left(B \rightarrow X_{s} \gamma\right)=(3.32 \pm$ $0.15) \times 10^{-4}[89,90]$ results in the allowed regions depicted in Fig. 14. Figure 14 shows just three examples of the allowed regions in the $\left(b_{L}-2 \lambda_{L}, b_{R}+2 \lambda_{R}\right)$ parameter space. These correspond to $p=0,0.7$, and 1.0 while $g^{\prime \prime}=18$. Again, the dependence on $g^{\prime \prime}$ can be ignored for our purpose. The allowed values are highly correlated. The $95 \%$ C.L. widths of the strips are about $0.005,0.007$, and 0.7 for $p=1,0.7$, and 0 , respectively. The allowed values of $b_{L}-2 \lambda_{L}$ range between about -30 and 30 when $p=1$. The compact formulation of the allowed values for the remaining parameters read $\left|p\left(b_{R}+2 \lambda_{R}\right)\right| \lesssim 0.3$.

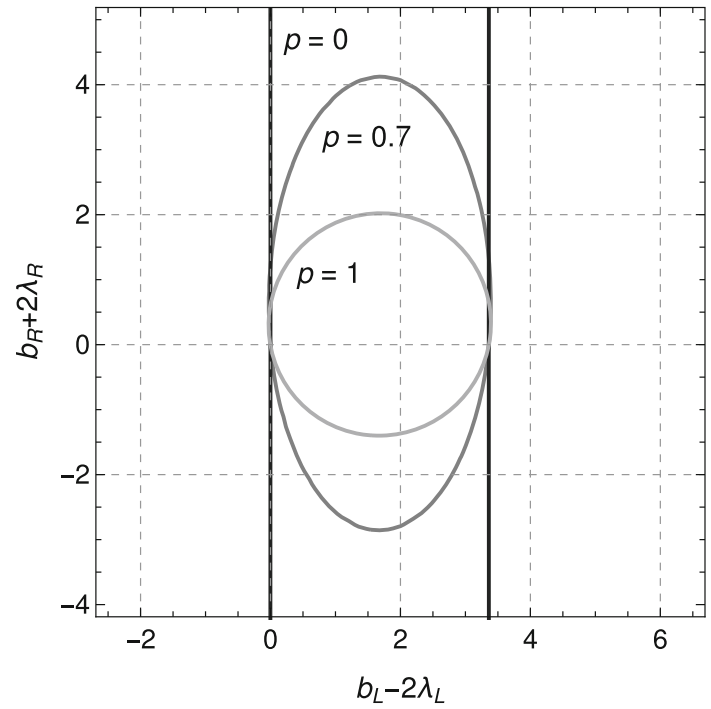

Fig. 13 The $95 \%$ C.L. $Z \rightarrow b \bar{b}$ allowed regions of the $b$ and $\lambda$ parameters when $g^{\prime \prime}=18$. The allowed regions form closed elliptical bands; the darkest gray correspond to $p=0$, the lightest to $p=1$, with $p=0.7$ in between. When $p=0$, only parts of two parallel bands of an "infinite" ellipse can be seen

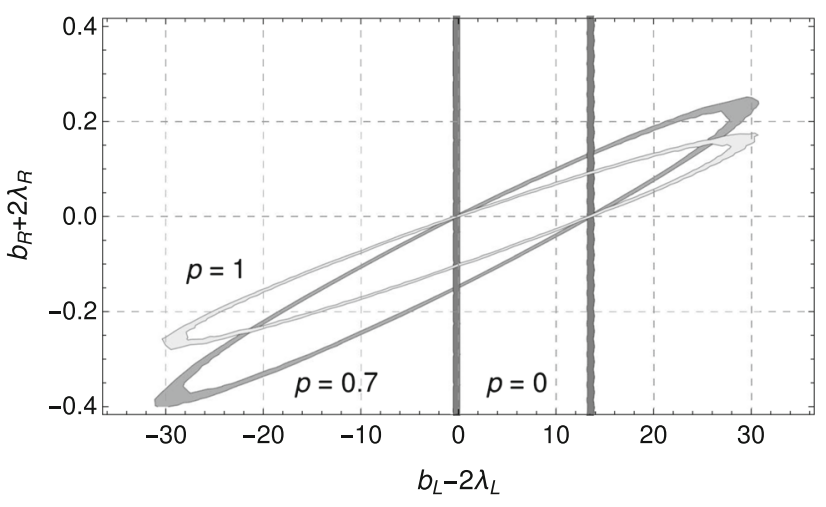

Fig. 14 The 95\% C.L. $B \rightarrow X_{s} \gamma$ allowed regions of the $b$ and $\lambda$ parameters when $g^{\prime \prime}=18$. The allowed regions form closed elliptical bands; the darkest gray correspond to $p=0$, the lightest to $p=1$, with $p=0.7$ in between. When $p=0$, only parts of two parallel bands of an "infinite" ellipse can be seen

The combination of the two sets of the allowed regions significantly reduces the limits. For example, when $p=1$ there are four distinct overlaps of the allowed regions resulting in the following restrictions. First, there is the allowed region that includes the no-direct interactions point: $\left|b_{L}-2 \lambda_{L}\right| \lesssim$ 0.01 and $\left|b_{R}+2 \lambda_{R}\right| \lesssim 0.004$. The second region is in the vicinity of $b_{L}-2 \lambda_{L} \approx 0.02$ and $b_{R}+2 \lambda_{R} \approx-0.10$. Further, when $b_{L}-2 \lambda_{L} \approx 3.34$ then $b_{R}+2 \lambda_{R} \approx-0.08$. Finally, there is an allowed region around $b_{L}-2 \lambda_{L} \approx 3.36$ and $b_{R}+2 \lambda_{R} \approx 0.025$.

There are no low-energy limits on the values of the $b$ and $\lambda$ parameters individually. Thus, in principle, $b$ 's and $\lambda$ 's can be tuned to any values if their sum/difference falls within the 
allowed interval. However, if one does not wish to admit a fine-tuning of the parameters, it is in place to add some ad hoc restriction; say, the absolute values of the $b_{L, R}$ or $\lambda_{L, R}$ parameters should not be greater than 10 times the size of the allowed interval for $b_{L, R} \mp 2 \lambda_{L, R}$. Of course, if we formulated the tBESS model with the simplifying assumption $\lambda_{L, R}=0$ right from the beginning then the quoted restrictions would apply directly to the direct interaction parameters $b_{L, R}$.

After discussing limits on the $b_{L, R}$ parameters obtained from the LHC upper bounds on $\sigma \times \mathrm{BR}$ at the end of the previous subsection, the text of the present subsection further demonstrates the complexity of the tBESS phenomenology and the role as well as the limitations of the flavor physics experiments in disentangling it. First of all, the presence of the $\lambda$ parameters weakens the restrictions that these measurements can put on the direct interactions. Secondly, a thorough study that would include other flavor physics processes could help eliminate some of the four regions allowed by the combination of the $\Gamma(Z \rightarrow b \bar{b})$ and $\operatorname{BR}\left(B \rightarrow X_{s} \gamma\right)$ measurements. Having said that, one can see that the flavor physics restrictions possess the potential to provide valuable input complementing the direct LHC limits. Considering the limited discussion of this subsection only, the restrictions shown here are more or less consistent with the limits obtained from the LHC upper bounds.

\subsection{An experimental update}

In this subsection, we provide a brief review on how the recently published experimental upper bounds on $\sigma_{\text {prod }} \times$ BR changed the mass exclusion limits calculated above. These latest upper bounds originate from the ongoing analyses of the data collected at the LHC experiments. While there have been about $139 \mathrm{fb}^{-1}$ of data recorded to the date the progress of their analysis lags behind. There are still new bounds being published that are even based on the 2016 dataset of $36 \mathrm{fb}^{-1}$.

As expected, even with the bigger integrated luminosity the $W W$ and $W Z$ are the only channels that provide restrictions on the tBESS production cross sections. In particular, the new upper bounds for the $W Z$ channel were published in $[80,86]$. The upper bounds were based on the $36 \mathrm{fb}^{-1}$ and $139 \mathrm{fb}^{-1}$ datasets, respectively. The new upper bounds for the $W W$ channel were published in $[82,86]$. The upper limits of the former paper were based on the $77 \mathrm{fb}^{-1}$ dataset. The new bounds in [86] apply to the mass range above $1.3 \mathrm{TeV}$ only. In combination with some of the previous upper bounds [68], these new experimental bounds result in new mass exclusion limits for the tBESS vector triplet.

To demonstrate the effect of the new upper bounds we update the mass exclusion limits for the scenarios presented in Tables 2, 4, and 5. Thus, Table 6 contains the updated mass exclusion limits when there are no direct interactions.
Table 6 The updated mass exclusion limits (MEL) for the tBESS vector resonance triplet without the direct interactions for various values of $g^{\prime \prime}$

\begin{tabular}{lllllll}
\hline$g^{\prime \prime}$ & $12-20$ & 21 & 22 & 23 & 24 & 25 \\
\hline$\Gamma_{\text {tot }} / M_{\rho}$ & $>0.40$ & 0.33 & 0.26 & 0.21 & 0.17 & 0.13 \\
MEL $(\mathrm{TeV})$ & $>3$ & 2.94 & 2.84 & 2.73 & 2.65 & $2.53^{a}$ \\
\hline
\end{tabular}

${ }^{a}$ The mass is also not excluded within $(1.24 ; 1.30) \mathrm{TeV}$

Table 7 The updated smallest tBESS resonance mass exclusion limits (MEL) within the interval $\left|b_{L=R}\right| \leq 1$ assuming $b_{L=R} \equiv b_{L}=b_{R}$ and $p=1$ for various values of $g^{\prime \prime}$. The second and third rows contain, respectively, the values of $b_{L=R}$ and of the resonance fatness $\Gamma_{\text {tot }} / M_{\rho}$ that correspond to the quoted mass exclusion limit

\begin{tabular}{llllllll}
\hline$g^{\prime \prime}$ & $12-19$ & 20 & 21 & 22 & 23 & 24 & 25 \\
\hline$b_{L=R} \times 10^{2}$ & & 8.6 & 7.6 & 6.7 & 5.9 & 4.8 & 4.0 \\
$\Gamma_{\text {tot }} / M_{\rho}$ & $>0.44$ & 0.41 & 0.32 & 0.25 & 0.20 & 0.15 & 0.12 \\
MEL $(\mathrm{TeV})$ & $>3$ & 2.96 & 2.86 & 2.74 & 2.65 & $2.52^{a}$ & $2.41^{b}$ \\
\hline
\end{tabular}

${ }^{a}$ The mass is also not excluded within $(1.11 ; 1.30) \mathrm{TeV}$

${ }^{b}$ The mass is also not excluded within $(1.03 ; 1.30) \mathrm{TeV}$

Table 8 The updated smallest tBESS resonance mass exclusion limits (MEL) within the interval $0 \leq p \leq 1$ assuming $b_{L}=0$ and $b_{R}=0.1$ for various values of $g^{\prime \prime}$. The second and third rows contain, respectively, the values of $p$ and of the resonance fatness that correspond to the quoted mass exclusion limit

\begin{tabular}{llllllll}
\hline$g^{\prime \prime}$ & $12-19$ & 20 & 21 & 22 & 23 & 24 & 25 \\
\hline$p$ & & 1 & 1 & 0.962 & 0.910 & 0.796 & 0.755 \\
$\Gamma_{\text {tot }} / M_{\rho}$ & $>0.44$ & 0.40 & 0.32 & 0.25 & 0.20 & 0.14 & 0.11 \\
MEL $(\mathrm{TeV})$ & $>3$ & 2.97 & 2.87 & 2.73 & 2.63 & 2.45 & 2.28 \\
\hline
\end{tabular}

The updated values for the scenarios of Tables 4 and 5 are shown in Tables 7 and 8, respectively. We can see that the mass exclusion limits have increased by more than $1 \mathrm{TeV}$. In addition, all corresponding fatnesses have surpassed the $10 \%$ mark which makes the presented conclusions less reliable. That is why we restrain ourselves from displaying the mass exclusion limits when they exceed $3 \mathrm{TeV}$ where the precision of the NWA calculations becomes very questionable.

\section{Conclusions}

Motivated by the absence of any signal of new particles beyond the SM in the LHC measurements, we have studied the mass exclusion limits for the hypothetical tBESS vector resonance triplet. The exclusion limits have been established utilizing the experimental upper bounds on the $s$-channel resonance production cross section times branching ratio provided by the ATLAS and CMS Collaborations for various decay channels. 
The tBESS resonance triplet represents a possible signature of a strongly-interacting extension of the SM. It has been introduced in the context of the phenomenological Lagrangian where, besides the composite $125 \mathrm{GeV}$ Higgs boson, the $S U(2)_{L+R}$ triplet of composite vector resonances is explicitly present. The vector resonance has been built into the Lagrangian employing the hidden local symmetry approach. In the tBESS model, the vector triplet interacts universally with all fermions due to the mixing between the electroweak gauge boson fields and the vector triplet. In addition, the direct couplings of the vector triplet to the top and bottom quarks have been introduced.

Fourteen vector resonance decay channels, for which the experimental upper bounds were available to the date, have been considered in our analysis. Of these, only the $W W$ and $W Z$ channels provide the mass exclusion limits for the tBESS vector triplet in both direct interaction scenarios.

The impact of the direct interactions on the mass exclusion limits has been contrasted with the no direct interaction case. As expected, the introduction of the specific direct interaction pattern to the model has made the analysis of the limits significantly more complex. Besides the emergence of new free parameters, the direct interactions have made the bottom quark partonic content of the proton significant for the neutral resonance production in the Drell-Yan mode. In fact, there are the parameter space regions where over $90 \%$ of the neutral resonance production is comprised of $b \bar{b} \rightarrow \rho^{0}$. Consequently, the sensitivity of $b \bar{b} \rightarrow \rho^{0}$ to the direct interaction couplings impacts all mass exclusion limits founded on the neutral resonance channels including the $W W$ one. The disregard of the bottom quark contents of the colliding protons would alter our results qualitatively. The contribution of the partonic charm and strange quarks to the neutral and charged vector resonance production is about 5-7\%.

The experimental upper bounds used in our analysis were derived with the narrow resonance qualification. Consistently, the model cross section predictions have been calculated in the narrow width approximation. As a rule of thumb we consider our analysis as reliable for the resonance fatness below $10 \%$. The results obtained for the resonances with the fatness above this mark must be considered with caution. This is an important issue in the case of the tBESS vector resonance triplet whose decay width grows significantly with its mass.

When there are no direct interactions the resonance mass exclusion limits range between 2.94 and $2.53 \mathrm{TeV}$ for $g^{\prime \prime}$ between 21 and 25, respectively. The respective resonance fatnesses range between 33 and 13\%. Unfortunately, these are already above the $10 \%$ rule of thumb. Thus, the quoted limits should be considered with caution. When $g^{\prime \prime} \leq 20$ the mass exclusions limit exceeds $3 \mathrm{TeV}$ and the corresponding fatness surpasses $40 \%$. Therefore, we do not even attempt to quote the particular exclusion limits obtained by the NWA calculations for $g^{\prime \prime} \leq 20$.

For the scenario with the direct interactions, the mass exclusion limits for two different sets of the parameter constraints have been studied. First, it has been assumed that the direct interactions are L-R and top-bottom universal, i.e., $b_{L}=b_{R}, p=1$. In the second case, it has been assumed that there are no direct interactions to the left top-bottom quark doublet $\left(b_{L}=0\right)$ and that the resonance couples strongly to the right top-bottom quark doublet $\left(b_{R}=0.1\right)$. The relative strength of the direct interactions to the top and bottom quark has been left as a free parameter. In both cases, the minimal mass exclusion limits, when varying the remaining free parameters, have been found. In the first case, the limit ranges from 2.96 to $2.41 \mathrm{TeV}$ for $g^{\prime \prime}=20$ and $g^{\prime \prime}=25$, respectively. The respective resonance fatnesses range between 41 and $12 \%$. In the second case, the mass exclusion limits for $g^{\prime \prime}$ between 20 and 25 range from 2.97 to $2.28 \mathrm{TeV}$, respectively. The respective resonance fatnesses range between 40 and $11 \%$. In both cases, the mass exclusion limits exceed $3 \mathrm{TeV}$ when $g^{\prime \prime} \leq 19$. The corresponding resonance fatnesses surpass $44 \%$.

The upper experimental bounds were also used to establish the restrictions on the $b_{L, R}$ parameters of the direct couplings of the vector resonance at a given mass. It was observed that it was impossible to formulate a universal conclusion about these restrictions since the obtained limits strongly depend on the choice of other model's parameters, namely the resonance mass and the top-bottom splitting factor $p$. As an illustration, when $g^{\prime \prime}=18$ and $M_{\rho}=1.75 \mathrm{TeV}$ the model was completely excluded for $p=1$. When $M_{\rho}=1.85 \mathrm{TeV}$ and $p=1$ then $\left|b_{L, R}\right|<0.05$ is allowed. When $p$ was lowered to 0.7 the $b_{R}$ restrictions got relaxed to about $\left|b_{R}\right|<0.12$ and no restrictions on $b_{R}$ occur when $p=0.4$. Assuming a simplified version of the tBESS model where $\lambda_{L, R}=0$ the combination of the $\mathrm{BR}\left(B \rightarrow X_{s} \gamma\right)$ and $\Gamma(Z \rightarrow b \bar{b})$ measurements provided complementary restrictions on $b_{L, R}$ alone. With certain qualifications regarding other model's parameter values, it can be concluded that these two kinds of restrictions on $b_{L, R}$ neither contradict nor overwhelm each other. There are non-trivial overlaps of the allowed regions in the parameter space. A more comprehensive analysis of how various flavor physics measurements restrict the direct interactions would be a worthwhile contribution to the effort of understanding of the hypothetical new vector resonances.

Acknowledgements We would like to thank P. Beneš, F. Blaschke, and F. Riva for useful discussions. The work was supported by the grant LTT17018 of the Ministry of Education, Youth and Sports of the Czech Republic and by the COST Action CA15108 "Connecting insights in fundamental physics”. M.G. was supported by the Slovak CERN Fund. We would also like to thank the Slovak Institute for Basic Research for their support. 
Data Availability Statement This manuscript has no associated data or the data will not be deposited. [Authors' comment: There are no data associated with the manuscript.]

Open Access This article is licensed under a Creative Commons Attribution 4.0 International License, which permits use, sharing, adaptation, distribution and reproduction in any medium or format, as long as you give appropriate credit to the original author(s) and the source, provide a link to the Creative Commons licence, and indicate if changes were made. The images or other third party material in this article are included in the article's Creative Commons licence, unless indicated otherwise in a credit line to the material. If material is not included in the article's Creative Commons licence and your intended use is not permitted by statutory regulation or exceeds the permitted use, you will need to obtain permission directly from the copyright holder. To view a copy of this licence, visit http://creativecomm ons.org/licenses/by/4.0/.

Funded by SCOAP ${ }^{3}$.

\section{Appendix A: The tBESS Lagrangian}

In this Appendix, we review the basic structure of the tBESS effective Lagrangian. Its phenomenology, relevant to this paper, was discussed in Sect. 2. The complete description and analysis of the Lagrangian can be found in [36-38].

The Lagrangian can be split in three parts

$\mathcal{L}=\mathcal{L}_{\mathrm{GB}}+\mathcal{L}_{\mathrm{ESB}}+\mathcal{L}_{\text {ferm }}$,

where $\mathcal{L}_{\mathrm{GB}}$ describes the gauge boson sector. It includes the $S U(2)_{\text {HLS }}$ triplet $\mathbf{V}_{\mu}$ representing the new vector resonance triplet. Further, $\mathcal{L}_{\mathrm{ESB}}$ is the scalar sector responsible for spontaneous breaking of the electroweak and hidden local symmetries, and $\mathcal{L}_{\text {ferm }}$ is the fermion Lagrangian of the model.

The first term of Eq. (A.1) reads

$$
\begin{aligned}
\mathcal{L}_{\mathrm{GB}}= & \frac{1}{2 g^{2}} \operatorname{Tr}\left(\boldsymbol{W}_{\mu \nu} \boldsymbol{W}^{\mu \nu}\right)+\frac{1}{2 g^{\prime 2}} \operatorname{Tr}\left(\boldsymbol{B}_{\mu \nu} \boldsymbol{B}^{\mu \nu}\right) \\
& +\frac{2}{g^{\prime \prime 2}} \operatorname{Tr}\left(\boldsymbol{V}_{\mu \nu} \boldsymbol{V}^{\mu \nu}\right),
\end{aligned}
$$

where the field strength tensors of the $S U(2)_{L} \times U(1)_{Y} \times$ $S U(2)_{\text {HLS }}$ gauge fields are defined as

$$
\begin{aligned}
\boldsymbol{W}_{\mu \nu} & =\partial_{\mu} \boldsymbol{W}_{\nu}-\partial_{\nu} \boldsymbol{W}_{\mu}+\left[\boldsymbol{W}_{\mu}, \boldsymbol{W}_{\nu}\right], \\
\boldsymbol{B}_{\mu \nu} & =\partial_{\mu} \boldsymbol{B}_{v}-\partial_{\nu} \boldsymbol{B}_{\mu}, \\
\boldsymbol{V}_{\mu \nu} & =\partial_{\mu} \boldsymbol{V}_{\nu}-\partial_{\nu} \boldsymbol{V}_{\mu}+\left[\boldsymbol{V}_{\mu}, \boldsymbol{V}_{\nu}\right],
\end{aligned}
$$

where $\boldsymbol{W}_{\mu}=i g W_{\mu}^{a} \tau^{a}, \boldsymbol{B}_{\mu}=i g^{\prime} B_{\mu} \tau^{3}$, and $\boldsymbol{V}_{\mu}=i \frac{g^{\prime \prime}}{2} V_{\mu}^{a} \tau^{a}$ with the gauge couplings $g, g^{\prime}$, and $g^{\prime \prime}$, respectively.

The ESB sector contains six unphysical real scalar fields, would-be Goldstone bosons of the model's spontaneous symmetry breaking. The six real scalar fields $\varphi_{L}^{a}(x), \varphi_{R}^{a}(x), \quad a=1,2,3$, are introduced as parameters of the $S U(2)_{L} \times S U(2)_{R}$ group elements in the expform $\xi\left(\varphi_{L, R}\right)=\exp \left(i \varphi_{L, R} \boldsymbol{\tau} / v\right) \in S U(2)_{L, R}$ where $\boldsymbol{\varphi}=$ $\left(\varphi^{1}, \varphi^{2}, \varphi^{3}\right)$. We express $\mathcal{L}_{\text {ESB }}$ as a sum of two terms,
$\mathcal{L}_{\mathrm{ESB}}=\mathcal{L}_{h}+\mathcal{L}_{\mathrm{hV}}$,

where $\mathcal{L}_{h}$ contains the kinetic and mass terms, and the selfinteractions of the Higgs boson. The interaction term

$$
\begin{aligned}
\mathcal{L}_{\mathrm{hV}}= & -v^{2}\left[\operatorname{Tr}\left(\bar{\omega}^{\perp}\right)^{2}\left(1+2 a_{V} \frac{h}{v}\right)\right. \\
& \left.+\alpha \operatorname{Tr}\left(\bar{\omega}^{\|}\right)^{2}\left(1+2 a_{\rho} \frac{h}{v}\right)\right]
\end{aligned}
$$

is responsible for the masses of all gauge bosons including the new vector triplet, and describes their interactions with the Higgs boson, which are parameterized by the free parameters $a_{V}$ and $a_{\rho}$.

The quantities $\bar{\omega}_{\mu}^{\|}$and $\bar{\omega}_{\mu}^{\perp}$ are, respectively, the $S U(2)_{L+R}$ parallel and perpendicular projections of the gauged MaurerCartan 1-form,

$\bar{\omega}_{\mu}^{\|}=\omega_{\mu}^{\|}+\frac{1}{2}\left(\xi_{L}^{\dagger} \boldsymbol{W}_{\mu} \xi_{L}+\xi_{R}^{\dagger} \boldsymbol{B}_{\mu} \xi_{R}\right)-\boldsymbol{V}_{\mu}$,

$\bar{\omega}_{\mu}^{\perp}=\omega_{\mu}^{\perp}+\frac{1}{2}\left(\xi_{L}^{\dagger} \boldsymbol{W}_{\mu} \xi_{L}-\xi_{R}^{\dagger} \boldsymbol{B}_{\mu} \xi_{R}\right)$,

where $\omega_{\mu}^{\|, \perp}=\left(\xi_{L}^{\dagger} \partial_{\mu} \xi_{L} \pm \xi_{R}^{\dagger} \partial_{\mu} \xi_{R}\right) / 2$

The fermion sector of the Lagrangian can be structured into three parts

$\mathcal{L}_{\text {ferm }}=\mathcal{L}_{\text {ferm }}^{\mathrm{SM}}+\mathcal{L}_{\text {ferm }}^{\text {scalar }}+\mathcal{L}_{(t, b)}^{\mathrm{tBESS}}$,

where $\mathcal{L}_{\text {ferm }}^{\mathrm{SM}}$ contains the SM interactions of fermions with the electroweak gauge bosons, $\mathcal{L}_{\text {ferm }}^{\text {scalar }}$ is about the interactions of the fermions with the scalar fields and includes the fermion masses. Finally, $\mathcal{L}_{(t, b)}^{\mathrm{tBESS}}$ describes the third generation quark direct interactions with the vector resonance. In addition, it contains symmetry-allowed non-SM interactions of the third quark generation with the EW gauge bosons parametrized by free parameters $\lambda_{L}$ and $\lambda_{R}$. Here, we show the new physics part of the $(t, b)$ Lagrangian in the unitary (physical) gauge,

$$
\begin{aligned}
\mathcal{L}_{(t, b)}^{\mathrm{tBES}}= & i b_{L} \bar{\psi}_{L}(\boldsymbol{V}-\boldsymbol{W}) \psi_{L} \\
& +i b_{R} \bar{\psi}_{R} P\left(\boldsymbol{V}-\boldsymbol{B}^{R 3}\right) P \psi_{R} \\
& +i \lambda_{L} \bar{\psi}_{L}\left(\boldsymbol{W}-\boldsymbol{B}^{R 3}\right) \psi_{L} \\
& +i \lambda_{R} \bar{\psi}_{R} P\left(\boldsymbol{W}-\boldsymbol{B}^{R 3}\right) P \psi_{R},
\end{aligned}
$$

where $\psi$ denotes the usual $S U$ (2) top-bottom doublet and the matrix $P=\operatorname{diag}(1, p)$ disentangles the direct interaction of the vector triplet with the right top quark from the interaction with the right bottom quark.

\section{References}

1. C. Grojean, Proc. Sci., EPS-HEP2009, 008 (2009)

2. R. Contino. arXiv:1005.4269

3. G. Panico, A. Wulzer, Lect. Notes Phys. 913, 1 (2016) 
4. A. Arbey, G. Cacciapaglia, H. Cai, A. Deandrea, S. Le Corre, F. Sannino, Phys. Rev. D 95, 015028 (2017)

5. G. Panico, A. Wulzer, JHEP 1109, 135 (2011). arXiv:1106.2719

6. R. Contino, D. Marzocca, D. Pappadopulo, R. Rattazzi, JHEP 1110, $081(2011)$

7. O. Matsedonskyi, G. Panico, A. Wulzer, JHEP 1301, 164 (2013)

8. S. Weinberg, Phys. Rev. D 19, 1277 (1979)

9. L. Susskind, Phys. Rev. D 20, 2619 (1979)

10. E. Farhi, L. Susskind, Phys. Rep. 74, 277 (1981)

11. S. Dimopoulos, L. Susskind, Nucl. Phys. B 155, 237 (1979)

12. E. Eichten, K.D. Lane, Phys. Lett. B 90, 125 (1980)

13. B. Holdom, Phys. Rev. D 24, 1441 (1981)

14. B. Holdom, Phys. Lett. B 150, 301 (1985)

15. K. Yamawaki, M. Bando, K-i Matumoto, Phys. Rev. Lett. 56, 1335 (1986)

16. T. Appelquist, D. Karabali, L.C.R. Wijewardhana, ibid. 57, 957 (1986)

17. T. Akiba, T. Yanagida, Phys. Lett. B 169, 432 (1986)

18. T. Appelquist, L.C.R. Wijewardhana, Phys. Rev. D 36, 568 (1987)

19. K. Lane, E. Eichten, Phys. Lett. B 222, 274 (1989)

20. H.S. Fukano, M. Kurachi, S. Matsuzaki, K. Terashi, K. Yamawaki, Phys. Lett. B 750, 259 (2015)

21. C.T. Hill, Phys. Lett. B 266, 419 (1991)

22. C.T. Hill, Phys. Lett. B B345, 483 (1995)

23. J.-Y. Lang, S.-Z. Jiang, Q. Wang, Phys. Rev. D 79, 015002 (2009)

24. N. Arkani-Hamed, S. Dimopoulos, G. Dvali, Phys. Lett. B 429, 263 (1998)

25. I. Antoniadis, N. Arkani-Hamed, S. Dimopoulos, G. Dvali, Phys. Lett. B B436, 257 (1998)

26. L. Randall, R. Sundrum, Phys. Rev. Lett. 83, 3370 (1999)

27. L. Randall, R. Sundrum, Phys. Rev. Lett. 83, 4690 (1999)

28. J.M. Maldacena, Adv. Theor. Math. Phys. 2, 231 (1998)

29. E. Witten, Adv. Theor. Math. Phys. 2, 253 (1998)

30. S.S. Gubser et al., Phys. Lett. B 428, 105 (1998)

31. N. Arkani-Hamed et al., JHEP 0108, 017 (2001)

32. R. Rattazzi et al., JHEP 0104, 021 (2001)

33. R.S. Chivukula, B. Coleppa, S. Chiara, E. Simmons, H.-J. He, M. Kurachi, M. Tanabashi, Phys. Rev. D 74, 075011 (2006)

34. E. Accomando, S. De Curtis, D. Dominici, L. Fedeli, Phys. Rev. D 79, 055020 (2009)

35. R. Contino, T. Kramer, M. Son, R. Sundrum, JHEP 0705, 074 (2007)

36. M. Gintner, J. Juráň, I. Melo, Phys. Rev. D 84, 035013 (2011)

37. M. Gintner, J. Juráň, Eur. Phys. J. C 73, 2577 (2013)

38. M. Gintner, J. Juráň, Eur. Phys. J. C 76, 651 (2016) (erratum, Eur. Phys. J. C 77, 6 (2017)). arXiv: 1608.00463

39. M. Gintner, J. Juráň, Acta Phys. Polon. B 48, 1383 (2017). arXiv: 1705.04806

40. T.L. Barklow et al., In: Proceedings of 1996 DPF/DPB Summer Study on New Directions For High-Energy Physics (Snowmass 96), Snowmass, Colorado, 25 Jun-12 Jul 1996, edited by D.G. Cassel, L. Trindle Gennari, R.H. Siemann (Stanford, CA, Stanford Linear Accelerator Center, 1997. 2v.), p.735. arXiv:hep-ph/9704217

41. T. Han, Int. J. Mod. Phys. A 23, 4107 (2008)

42. T. Han, G. Valencia, Y. Wang, Phys. Rev. D 70, 034002 (2004)

43. X.-G. He, G. Valencia, Phys. Rev. D 66, 013004 (2002)

44. R. Contino, Y. Nomura, A. Pomarol, Nucl. Phys. B 671, 148 (2003)

45. K. Agashe, R. Contino, A. Pomarol, Nucl. Phys. B B719, 165 (2005)

46. B. Bellazzini, C. Csáki, J. Hubisz, J. Serra, J. Terning, JHEP 1211, 003 (2012). arXiv: 1205.4032

47. D. Liu, L.-T. Wang, K.-P. Xie, Phys. Rev. D 100, 075021 (2019). arXiv:1901.01674

48. D.B. Kaplan, Nucl. Phys. B 365, 259 (1991)

49. T. Han, Y.J. Kim, A. Likhoded, G. Valencia, Nucl. Phys. B 593, $415(2001)$
50. T. Han, D.L. Rainwater, G. Valencia, Phys. Rev. D 68, 015003 (2003)

51. E. Accomando, L. Fedeli, S. Moretti, S. De Curtis, D. Dominici, Phys. Rev. D 86, 115006 (2012)

52. L. Fedeli, S. Moretti, S. De Curtis, D. Dominici, Phys. Rev. D 87, 015021 (2013)

53. A.E.C. Hernández, R. Torre, Nucl. Phys. B 841, 188 (2010)

54. A.E.C. Hernández, B.D. Sáez, C.O. Dib, A. Zerwekh, Phys. Rev. D 96, 115027 (2017). arXiv:1707.05195

55. R.S. Chivukula, P. Ittisamai, K. Mohan, E.H. Simmons, Phys. Rev. D 96, 055043 (2017). arXiv:1707.01080

56. M. Bando, T. Kugo, K. Yamawaki, Phys. Rep. 164, 217 (1988)

57. R. Casalbuoni, S. De Curtis, D. Dominici, R. Gatto, Phys. Lett. 155B, 95 (1985)

58. R. Casalbuoni, S. De Curtis, D. Dominici, R. Gatto, Nucl. Phys. B 282, 235 (1987)

59. R. Casalbuoni, P. Chiappetta, S. De Curtis, F. Feruglio, R. Gatto, B. Mele, J. Terron, Phys. Lett. B 249, 130 (1990)

60. D. Pappadopulo, A. Thamm, R. Torre, A. Wulzer, JHEP 1409, 060 (2014). arXiv:1402.4431

61. S. Dawson, Nucl. Phys. B 249, 42 (1985)

62. D.B. Clark, E. Godat, F.I. Olness, arXiv:1605.08012. Mane Parse package download: https://ncteq.hepforge.org/mma/index.html

63. A. Buckley et al., Eur. Phys. J. C 75, 132 (2015). arXiv:1412.7420. LHAPDF6 PDFs download: http://lhapdf.hepforge.org/pdfsets

64. ATLAS Collaboration, ATLAS-CONF-EXOT-2017-31. https://atlas.web.cern.ch/Atlas/GROUPS/PHYSICS/PAPERS/ EXOT-2017-31/

65. ATLAS Collaboration, JHEP 1803 (2018) 009; arXiv:1708.09638

66. CMS Collaboration, CMS-PAS-B2G-17-001

67. ATLAS Collaboration, ATLAS-CONF-2016-082

68. ATLAS Collaboration, ATLAS-CONF-2016-062

69. ATLAS Collaboration, JHEP 1803 (2018) 174; arXiv: 1712.06518

70. ATLAS Collaboration, Phys. Rev. D 96, 052004 (2017); arXiv:1703.09127

71. CMS Collaboration, CMS-PAS-EXO-16-056

72. ATLAS Collaboration, ATLAS-CONF-2017-027

73. ATLAS Collaboration, ATLAS-CONF-2017-016

74. ATLAS Collaboration, JHEP 1801 (2018) 055. arXiv:1709.07242

75. ATLAS Collaboration, Phys. Rev. Lett. 120, 161802 (2018). arXiv: 1801.06992

76. CMS Collaboration, JHEP 1904 (2019) 031. arXiv:1810.05905

77. ATLAS Collaboration, Phys. Rev. D 98, 032016 (2018). arXiv: 1805.09299

78. ATLAS Collaboration, Phys. Lett. B788, 347 (2019). arXiv: 1807.10473

79. CMS Collaboration, CMS-PAS-B2G-17-010

80. CMS Collaboration, CMS-PAS-B2G-18-006

81. C.M.S. Collaboration, Phys. Lett. B 792, 107 (2019). arXiv: 1807.11421

82. CMS Collaboration, CMS-PAS-B2G-18-002

83. ATLAS Collaboration, ATLAS-CONF-2018-017

84. ATLAS Collaboration, ATLAS-CONF-2019-007

85. ATLAS Collaboration, ATLAS-CONF-2019-001

86. ATLAS Collaboration, ATLAS-CONF-2019-003

87. R.L. Delgado, A. Dobado, D. Espriu, C. Garcia-Garcia, M.J. Herrero, X. Marcano, J.J. Sanz-Cillero, JHEP 1711, 098 (2017). arXiv: 1707.04580

88. R.L. Delgado, C. Garcia-Garcia, M.J. Herrero, JHEP 1911, 065 (2019). arXiv:1907.11957

89. M. Tanabashi et al., Particle Data Group. Phys. Rev. D 98, 030001 (2018)

90. Y. Amhis et al. (Heavy Flavor Averaging Group). arXiv:1909.12524 